hep-th/0504090

FIAN/TD/07-05

\title{
Actions, Charges and Off-Shell Fields in the Unfolded Dynamics Approach
}

\author{
M.A. Vasiliev \\ I.E.Tamm Department of Theoretical Physics, Lebedev Physical Institute, \\ Leninsky prospect 53, 119991, Moscow, Russia
}

\begin{abstract}
Within unfolded dynamics approach, we represent actions and conserved charges as elements of cohomology of the $L_{\infty}$ algebra underlying the unfolded formulation of a given dynamical system. The unfolded off-shell constraints for symmetric fields of all spins in Minkowski space are shown to have the form of zero curvature and covariant constancy conditions for 1-forms and 0-forms taking values in an appropriate star product algebra. Unfolded formulation of Yang-Mills and Einstein equations is presented in a closed form.
\end{abstract}

\section{Introduction}

Nonlinear dynamics of massless higher spin (HS) gauge fields was formulated $[1,2]$ within the unfolded formulation [3] in which the dynamical equations have a form of certain covariant constancy and zero-curvature conditions with respect to space-time coordinates. This form of the equations is useful in various respects because its formal consistency controls gauge symmetries and diffeomorphisms. The unfolded formalism works beautifully and efficiently for the infinite sets of fields of all spins through the concise formalism of certain generating functions $[1,2]$. Although the unfolded formulation exists, in principle, for any dynamical system, it may not necessarily be clear how to develop 
it in a closed form for one or another specific model. In particular, the unfolded formulation of the nonlinear Einstein and Yang-Mills equations was not known so far, although some first order corrections have been explicitly found in [4] for pure Einstein gravity. One of the motivations of this paper was to fill in this gap by constructing unfolded formulation for these nonlinear lower spin systems.

One has to distinguish between off-shell unfolded formulation that only takes into account the Bianchi identities and on-shell formulation that takes into account field equations. The formulation of the HS dynamics in $A d S_{d}$ developed in [2] gives both the off-shell formulation (as was emphasized in [5]) and on-shell formulation. The latter results from factorization of the ideal associated with the off-shell degrees of freedom that are trivial on-mass-shell [2] (for more details see [6]). As shown originally in [7], consistent HS interactions with gravity require $A d S$ background with non-zero cosmological constant as the most symmetric vacuum. This is why the formalism of [2] was designed to incorporate $A d S$ background and $A d S$ symmetries in a most natural way.

The relevance of the $A d S$ background concerns the action principle and, therefore, HS field equations. There is no reason, however, to expect that $A d S$ description may play any significant role in the off-shell formulation. Indeed, Bianchi identities in any system are merely identities: no obstruction can result from rewriting them in the unfolded form in any background. Therefore, the unfolded off-shell formulation of [2] should be expected to admit a flat limit in one or another way. A new result presented in this paper is the unfolded form of off-shell nonlinear constraints for symmetric fields of all spins in flat background.

So far the unfolded formulation was applied [3] (see also [8, 9, 6] for reviews) to the analysis of the field equations and/or constraints. In this paper we extend this approach to the action level. The explanation of the general idea and main ingredients of the unfolded formulation is the content of section 2 . Let us note that a closely related structure is $L_{\infty}$ algebra [10].

The unfolded form of the off-shell constraints for symmetric fields turns out to be so simple and inspiring that we start in section 3 with giving the final results leaving detailed proofs and explanations for the rest of the paper which is organized as follows. After recalling unfolded formulation of free symmetric fields in section 4 and a few relevant algebraic properties in section 5 we show in section 6 that linearization of the nonlinear equations of section 3 correctly reproduces the linearized unfolded constraints for massless fields. Nonlinear unfolded constraints are analysed in section 7. In particular, in subsection 7.1 the unfolded formulation of the off-shell constraints for Yang-Mills and 
Einstein theories is obtained in the closed form. The on-shell unfolded formulation for Yang-Mills and Einstein equations is discussed in section 8. It is shown here that the key constraints still have the form of Yang-Mills and Einstein equations although in terms of different variables. Alternative versions of unfolded constraints are discussed in section 9. In Appendix A we extend the construction of unfolded dynamics to the general case with explicit dependence on space-time coordinates. Theorems on $\sigma_{-}$cohomology, which play central role in the dynamical analysis of an unfolded system, are reproduced in Appendix B to make this paper as self contained as possible.

\section{Unfolded Dynamics}

\subsection{Unfolded equations}

Let $M^{d}$ be a $d$-dimensional space-time manifold with coordinates $x^{\underline{n}}(\underline{n}=$ $0,1, \ldots d-1)$. By unfolded formulation of a linear or nonlinear system of differential equations and/or constraints in $M^{d}$ we mean its equivalent reformulation in the first-order form

$$
d W^{\alpha}(x)=G^{\alpha}(W(x))
$$

where $d=d x^{\underline{n}} \frac{\partial}{\partial x^{\underline{n}}}$ is the de Rham differential in $M^{d}, W^{\alpha}(x)$ is a set of degree $p_{\alpha}$ differential forms and $G^{\alpha}(W)$ is some degree $p_{\alpha}+1$ function of the differential forms $W^{\alpha}$

$$
G^{\alpha}(W)=\sum_{n=1}^{\infty} f^{\alpha}{ }_{\beta_{1} \ldots \beta_{n}} W^{\beta_{1}} \wedge \ldots \wedge W^{\beta_{n}},
$$

where the coefficients $f^{\alpha}{ }_{\beta_{1} \ldots \beta_{n}}$ satisfy the (anti)symmetry condition

$$
f^{\alpha}{ }_{\beta_{1} \ldots \beta_{k} \ldots \beta_{l} \ldots \beta_{n}}=(-1)^{p_{\beta_{k}} p_{\beta_{l}}} f^{\alpha}{ }_{\beta_{1} \ldots \beta_{l} \ldots \beta_{k} \ldots \beta_{n}}
$$

(an extension to the supersymmetric case with addition boson-fermion grading is straightforward) and $G^{\alpha}$ satisfies the condition

$$
G^{\beta}(W) \wedge \frac{\partial G^{\alpha}(W)}{\partial W^{\beta}}=0
$$

equivalent to the following generalized Jacobi identity on the structure coefficients

$$
\sum_{n=0}^{m}(n+1) f_{\left[\beta_{1} \ldots \beta_{m-n}\right.}^{\gamma} f_{\left.\gamma \beta_{m-n+1} \ldots \beta_{m}\right\}}^{\alpha}=0,
$$


where the brackets [ $\}$ denote an appropriate (anti)symmetrization of all indices $\beta_{i}$. Strictly speaking, the generalized Jacobi identities (2.5) have to be satisfied only at $p_{\alpha}<d$ for the case of a $d$-dimensional manifold $\mathcal{M}^{d}$ where any $d+1$ form is zero. Given solution of (2.5) it defines a free differential algebra. We call a free differential algebra universal if the generalized Jacobi identity is true for all values of indices, i.e., independently of a particular value of space-time dimension. The HS free differential algebras discussed in this paper belong to the universal class. Note that every universal free differential algebra is associated with some $L_{\infty}$ algebra $^{1}$ [10].

The condition (2.4), which can equivalently be rewritten as

$$
Q^{2}=0, \quad Q=G^{\alpha}(W) \frac{\partial}{\partial W^{\alpha}},
$$

guarantees the formal consistency of the unfolded system (2.1) with $d^{2}=0$ for all $W^{\alpha}$. This form of the compatibility condition manifests close relation of our construction with $Q$-manifolds [14] and, more generally, with the Batalin-Vilkovisky approach [15] (see [16] for a recent discussion of the latter relationship). The unfolded equations (2.1) now imply

$$
d F(W(x))=Q(F(W(x))
$$

for any function $F(W)$ that does not contain explicit dependence on the coordinates $x^{\underline{n}}$, i.e., depends only on $W^{\alpha}$. This form of the unfolded equations is analogous to the Hamiltonian equations in the standard 1d Hamiltonian dynamics. (For completeness, let us mention that, as explained in more detail in Appendix A, the unfolded formulation admits a natural extension to the case where the function $G^{\alpha}=G^{\alpha}(W, x)$ depends explicitly on the coordinates $x^{\underline{n}}$.)

The equation (2.1) is invariant under the gauge transformation

$$
\delta W^{\alpha}=d \varepsilon^{\alpha}+\varepsilon^{\beta} \frac{\partial G^{\alpha}(W)}{\partial W^{\beta}},
$$

\footnotetext{
${ }^{1}$ The difference is that a form degree $p_{\alpha}$ of $W^{\alpha}$ is fixed in a universal free differential algebra while $W^{\alpha}$ in $L_{\infty}$ are treated as local coordinates of a graded manifold. A universal free differential algebra can be obtained from a $L_{\infty}$ algebra by an appropriate projection to specific form degrees. (For more detail on this relationship we refer the reader to [11]. I am grateful to Maxim Grigoriev for illuminating discussion of this relationship.) The language of free differential algebras used in the earlier papers [3, 9] (however, with the important extension to the case with 0-forms included), where the unfolding approach was originally suggested and applied to the analysis of HS models, was introduced into field-theoretical literature in [12]. In the absence of 0-forms, the structure of these algebras was classified by Sullivan [13].
} 
where the derivative $\frac{\partial}{\partial W^{\beta}}$ is left and the gauge parameter $\varepsilon^{\alpha}(x)$ is a $\left(p_{\alpha}-1\right)$ form. (0-forms $W^{\alpha}$ do not give rise to gauge parameters.)

An example of a free differential algebra can be constructed as follows. Let $h$ be a Lie algebra, a basis of which is the set $\left\{T_{\alpha}\right\}$. Let $\omega=\omega^{\alpha} T_{\alpha}$ be a 1-form taking values in $h$. If one chooses $G(\omega)=-\omega \wedge \omega \equiv-\frac{1}{2} \omega^{\alpha} \wedge \omega^{\beta}\left[T_{\alpha}, T_{\beta}\right]$, then the equation (2.1) with $W=\omega$ is the zero-curvature equation

$$
d \omega+\omega \wedge \omega=0 .
$$

The relation (2.4) amounts to the usual Jacobi identity for the Lie algebra $h$. (2.8) is then the usual gauge transformation of the connection $\omega$.

If the set $W^{\alpha}$ also contains some $p$-forms denoted by $\mathcal{C}^{i}$ (e.g. 0 -forms) and if the functions $G^{i}$ are linear in $\omega$ and $C$

$$
G^{i}=-\omega^{\alpha}\left(T_{\alpha}\right)_{j}^{i} \wedge \mathcal{C}^{j}
$$

then the relation (2.4) implies that the matrices $\left(T_{\alpha}\right)^{i}{ }_{j}$ form some representation $T$ of $h$, acting in a space $V$ where the $\mathcal{C}^{i}$ take their values. The corresponding equation (2.1) is a covariant constancy condition

$$
D_{\omega} \mathcal{C}=0
$$

with $D_{\omega} \equiv d+\omega$ being a covariant derivative in the $h$-module $V$.

The zero-curvature equations (2.9) usually describe background geometry in a coordinate independent way. For example, let $h$ be the Poincare algebra with the gauge fields

$$
\omega(x)=e^{n}(x) P_{n}+\omega^{n m}(x) L_{n m},
$$

where $P_{n}$ and $L_{n m}$ are generators of translations and Lorentz transformations with the respective gauge fields $e^{n}(x)$ and $\omega^{n m}(x)$ to be identified with the frame 1-form and Lorentz connection, respectively (fiber Lorentz vector indices $m, n \ldots$ run from 0 to $d-1$ and are raised and lowered by the flat Minkowski metric). It is well-known that the zero-curvature condition (2.9) for the Poincarè algebra amounts to the zero-torsion condition

$$
R^{n}=d e^{n}-\omega_{m}^{n} \wedge e^{m}=0
$$

which expresses $\omega^{n}{ }_{m}$ in terms of derivatives of the frame field, and the Riemann tensor vanishing condition

$$
R^{m n}=d \omega^{m n}-\omega^{m}{ }_{k} \wedge \omega^{k n}=0,
$$


which implies flat Minkowski space-time geometry.

As a result, at the condition that $e_{0}{ }^{n}(x)$ is a nondegenerate matrix, the zero-curvature condition (2.9) for the Poincarè algebra describes Minkowski space-time in a coordinate independent way. By choosing a different Lie algebra $h$ one can describe a different background like, e.g., (anti-) de Sitter. The covariant constancy equation (2.11) can describe linear equations in a chosen background.

\subsection{Dynamical content}

Any consistent system of partial differential equations and/or constraints can, in principle, be re-written in the unfolded form by adding enough auxiliary variables [17]. The unfolded formulation is a multidimensional covariant (i.e. coordinate independent) generalization of the first-order formulation

$$
d t \frac{\partial}{\partial t} q^{\alpha}=G^{\alpha}(q), \quad G^{\alpha}=e F^{\alpha}
$$

available for any system of ordinary differential equations by adding auxiliary variables to be identified with higher derivatives of the dynamical variables of the original system of differential equations. Here $e$ is a einbein 1-form that can be identified with $d t$ because a one-dimensional space-time is always flat. In this $d=1$ example, the condition (2.4) trivializes because $e \wedge e=0$, i.e. any function $F^{\alpha}(q)$ is allowed. Note that this condition is true for an arbitrary number of coordinates of the ambient space (i.e., $d x \underline{\underline{m}}$ ), provided that $e=d x \underline{\underline{m}} e_{\underline{m}}$ does not carry fiber indices. This means that the system is universal. Although, for the case of $d>1$ with $e^{n}$ having $d$ components, nontrivial consistency conditions have to be taken into account, the simplest $d=1$ example illustrates the general mechanism.

The structure of first-order ordinary differential equations is as follows

$$
\frac{\partial}{\partial t} q_{i}^{\tilde{\alpha}}=a_{i}^{\tilde{\alpha}} q_{i+1}^{\tilde{\alpha}}+\ldots, \quad i=0,1,2, \ldots
$$

where $a_{i}^{\tilde{\alpha}}$ are some coefficients and dots denote higher order nonlinear corrections. If all coefficients $a_{i}^{\tilde{\alpha}}$ are different from zero, the equations (2.16), treated perturbatively, describe a set of constraints that express all $q_{i+1}^{\tilde{\alpha}}$ via derivatives of $q_{0}^{\tilde{\alpha}}$. If some coefficient $a_{j}^{\tilde{\alpha}}$ vanishes, this means that there is some nontrivial differential equation on $q_{0}^{\tilde{\alpha}}$, which is of order $j$ at the linearized level. In the first order formulation (in particular, in the Hamiltonian formalism) the initial data problem is fixed in terms of values of all variables $q^{\alpha}$ at a given point of "space-time" $t=t_{0}$. 
In the general case of $d>1$ these properties have clear analogues. Nontrivial dynamical fields (i.e., those that are different from auxiliary fields expressed via derivatives of the dynamical fields), gauge symmetries and true differential field equations, are classified in terms of the so-called $\sigma_{-}$cohomology [18] that roughly speaking controls zeros among the coefficients analogous to $a_{i}^{\tilde{\alpha}}$ of the linearized equations. The $\sigma_{-}$cohomology is a perturbative concept that emerges in the linearized analysis with

$$
W^{\alpha}(x)=W_{0}^{\alpha}(x)+W_{1}^{\alpha}(x),
$$

where $W_{0}^{\alpha}(x)$ is a particular solution of $(2.1)$ and $W_{1}^{\alpha}(x)$ is treated as a perturbation. $W_{0}^{\alpha}(x)$ is nonzero in a field-theoretical system because it should describe a background gravitational field. Typically, $W_{0}^{\alpha}(x)=\left(e_{0}^{n}(x), \omega_{0}^{n m}(x)\right)$, where $e_{0}^{n}(x)$ and $\omega_{0}^{n m}(x)$ are frame and Lorentz connection 1-forms of the background space-time (e.g., Minkowski, $(A) d S$, etc.) Linearized equations (2.1)

$$
d W_{1}^{\alpha}(x)=\left.W_{1}^{\beta}(x) \frac{\delta G^{\alpha}}{\delta W^{\beta}}\right|_{W=W_{0}}
$$

can be rewritten in the form (2.11)

$$
D_{0} W_{1}^{\alpha}(x)=0,
$$

where $D_{0}$ is some differential which squares to zero as a consequence of the consistency condition (2.4),

$$
D_{0}^{2}=0 .
$$

Usually a set of fields $W_{1}$ admits a grading $G$ with the spectrum bounded from below. The grading $G$ typically counts a rank of a tensor (equivalently, a power of an appropriate generating polynomial). Suppose that

$$
D_{0}=\tilde{D}_{0}+\sigma_{-}+\sigma_{+},
$$

where

$$
\left[G, \sigma_{-}\right]=-\sigma_{-}, \quad\left[G, \tilde{D}_{0}\right]=0
$$

and $\sigma_{+}$is a sum of operators of positive grade. From (2.20) it follows that

$$
\sigma_{-}^{2}=0 .
$$

Provided that $\sigma_{-}$acts vertically (i.e. does not differentiate $x^{\underline{n}}$ ), the dynamical content of the dynamical system under investigation is determined by cohomology of $\sigma_{-}$. Namely, as shown in [18], for a $p_{\alpha}$-form $W^{\alpha}$ that takes values 
in a vector space $V, H^{p+1}\left(\sigma_{-}, V\right), H^{p}\left(\sigma_{-}, V\right)$ and $H^{p-1}\left(\sigma_{-}, V\right)$ describe, respectively, differential equations, dynamical fields and differential gauge symmetries encoded by the equation (2.19). (For more detail see Appendix B and [6].) The case with $H^{p+1}\left(\sigma_{-}, V\right)=0$ is analogous to that of (2.16) with all coefficients $a_{i}^{\tilde{\alpha}}$ different from zero where no differential equations on the dynamical variables are imposed. In this case, the equations (2.1) just express the Bianchi identities for the constraints on auxiliary fields. Equations of this type will be referred to as off-shell. (Let us stress that this definition is true both for linear and for non-linear cases: nonlinear equations are off-shell if their linearization is off-shell.)

The degrees of freedom, that is variables that fix a (local) solution of the equations (2.1) modulo gauge ambiguity, are values of all 0-forms $C^{\phi}(x)$ among $W^{\alpha}(x)$, taken at any given point $x^{\underline{n}}=x_{0}^{\underline{n}}[9]$ analogous to $t_{0}$ of the $d=1$ case. For a field-theoretical system with infinite number of degrees of freedom to be described this way an infinite set of 0 -forms has to be introduced. For a topological system with a finite number of degrees of freedom, unfolded equations with a finite number of 0 -forms may be available.

Whether a system is on-shell or off-shell depends not only on $G^{\alpha}(W)$ but, in first place, on a space-time $M^{d}$ and a chosen vacuum solution $W_{0}^{\alpha}(x)$. In particular, dynamical interpretation may be different for different dimensions of the space-time. On the other hand, from the unfolded equations (2.1) it follows that the dependence on the coordinates $x^{\underline{n}}$ is reconstructed in terms of values of the fields $W^{\alpha}(x)$ at any given point $x_{0}^{n}$. This means, in turn, that the role of coordinates $x^{\underline{n}}$ is somewhat auxiliary as was first noted in the context of HS dynamics in [19]. As emphasized in [20] the role of coordinates is that they help to visualize physical local events via a specific physical process. Moreover, a result of such a visualization to large extent depends not on a chosen manifold $M^{d}$, where one or another equation (2.1) is written, but mostly on the specific form of the function $G^{\alpha}$. This property was used for practical purposes of equivalent reformulation of field equations in different space-times with additional commuting [21, 20] and anticommuting [22] coordinates.

\subsection{Actions and conserved charges}

Independently of the dynamical interpretation of a given unfolded system, its invariants like actions and conserved charges turn out to be associated with the cohomology of the differential $Q$ (2.6).

First suppose that the system (2.1) is off-shell. Let us extend the set of forms $W^{\alpha}$ with a $d$-1-form $E$ and a $d$-form $L$ extending the equations (2.1) 
with the equations

$$
\begin{gathered}
d E=L-\mathcal{L}(W), \\
d L=0,
\end{gathered}
$$

where $\mathcal{L}(W)$ is some Lagrangian function of the variables $W^{\alpha}$ which is $Q$-closed

$$
Q \mathcal{L}=0: \quad G^{\alpha}(W) \frac{\partial}{\partial W^{\alpha}} \mathcal{L}(W)=0 .
$$

The latter condition guarantees that the extended system remains consistent, i.e. $Q^{\prime}=Q+(L-\mathcal{L}) \frac{\partial}{\partial E}$ satisfies $\left(Q^{\prime}\right)^{2}=0$.

We define action $S$ as the integral of $L$ over a $d$-cycle $M^{d}$

$$
S=\int_{M^{d}} L,
$$

which, in turn, can be embedded into a larger space. By (2.25), the result is independent of local variations of this embedding. The action $S(2.27)$ is gauge invariant. Actually, the gauge transformation (2.8) gives for $E$ and $L$

$$
\begin{gathered}
\delta E=d \epsilon_{E}+\epsilon_{L}-\epsilon^{\alpha} \frac{\partial \mathcal{L}(W)}{\partial W^{\alpha}}, \\
\delta L=d \epsilon_{L} .
\end{gathered}
$$

Assuming that $M^{d}$ has no boundary (or that the field variables fall down fast enough at infinity) the action (2.27) remains invariant under the transformation (2.29). Therefore, the action (2.27) is invariant under the full set of the gauge transformations (2.8).

One can use the gauge parameter $\epsilon_{L}$ to gauge fix $E$ to zero. In this gauge, the action (2.27) takes the form

$$
S=\int_{M^{d}} \mathcal{L}(W) .
$$

Taking into account (2.26) it is easy to see directly that this action is invariant under the gauge transformations (2.8).

If $\mathcal{L}$ is $Q$-exact, i.e. $\mathcal{L}=G^{\alpha}(W) \frac{\partial}{\partial W^{\alpha}} \mathcal{E}$, by a field redefinition

$$
E \rightarrow E^{\prime}-\mathcal{E}(W),
$$

this case is equivalent to that with $\mathcal{L}=0$, i.e. $Q$-exact Lagrangians $\mathcal{L}(W)$ do not generate nontrivial actions (as is also obvious from (2.7)). Thus, nontrivial invariant actions are in the one-to-one correspondence with the $Q$ cohomology 
of the original off-shell system. A Lagrangian function $\mathcal{L}$ is a representative of a $Q$ cohomology class.

If the system is on-shell and a representative $\mathcal{L}$ of the $Q$ cohomology is a $p$-form, the same formula (2.30) describes a conserved charge as an integral over a $p$-cycle $\Sigma$

$$
q=\int_{\Sigma} \mathcal{L}
$$

Taking into account that whether a dynamical system is on-shell or offshell depends on the choice of space-time manifold $M^{d}$ rather than on $G^{\alpha}(W)$, we conclude that there is no big difference in the unfolded dynamics approach between actions and conserved charges associated with the same $Q$-cohomology in seemingly different dynamical systems described by the same operator $Q$. It is interesting to study physical consequences of this surprising identification.

Another point we would like to stress is that the $Q$-cohomology describes full invariant actions and charges of the unfolded system at hand rather than perturbative deformations of interactions, as one would normally expect of a cohomology.

\subsection{Scalar field example}

To describe an off-shell scalar field $C(x)$ in the unfolded form, we introduce following [18] the infinite set of 0 -forms $C_{m_{1} \ldots m_{n}}(x)(n=0,1,2, \ldots)$, which are completely symmetric tensors

$$
C_{m_{1} \ldots m_{n}}=C_{\left\{m_{1} \ldots m_{n}\right\}}
$$

The off-shell "unfolded" equations are

$$
d C_{m_{1} \ldots m_{n}}=e_{0}^{k} C_{m_{1} \ldots m_{n} k}, \quad(n=0,1, \ldots),
$$

where we use Cartesian coordinates with vanishing Lorentz connection that allows us to replace the Lorentz covariant derivative $D_{0}^{L}$ by the exterior differential $d$. This system is formally consistent because applying $d$ on both sides of (2.34) does not lead to any new condition as $e_{0}^{k} \wedge e_{0}^{l}=-e_{0}^{l} \wedge e_{0}^{k}$. This property implies that the space $V$ of 0 -forms $C_{m_{1} \ldots m_{n}}$ spans some representation of the Poincaré algebra $i s o(d-1,1)$. In other words, $V$ is an infinite-dimensional iso $(d-1,1)$-module ${ }^{2}$.

\footnotetext{
${ }^{2}$ Strictly speaking, to apply the general argument of subsection 2.1 one has to check that the equation remains consistent for any flat connection in $i s o(d-1,1)$. It is not hard to see that this is true indeed.
} 
Let us identify the scalar field $C(x)$ with $C_{m_{1} \ldots m_{n}}(x)$ at $n=0$. Then the first two equations of the system (2.34) read

$$
\partial_{\underline{n}} C=C_{\underline{n}}, \quad \partial_{\underline{n}} C_{\underline{m}}=C_{\underline{m n}},
$$

where we have identified the world and tangent indices via $\left(e_{0}\right)_{\underline{m}}^{m}=\delta_{\underline{m}}^{m}$. The first of these equations just tells us that $C_{\underline{n}}$ is the first derivative of $\bar{C}$. The second one tells us that $C_{\underline{n} \underline{m}}$ is the second derivative of $C$. All other equations in (2.34) express highest tensors in terms of the higher-order derivatives

$$
C_{\underline{m}_{1} \ldots \underline{m}_{n}}=\partial_{\underline{m}_{1}} \ldots \partial_{\underline{m}_{n}} C
$$

and impose no conditions on $C$. From this formula it is clear that the meaning of the 0-forms $C_{\underline{n}_{1} \ldots \underline{n}_{n}}$ is that they form a basis in the space of all derivatives of the dynamical field $C(x)$, including the derivative of order zero which is the field $C(x)$ itself. Thus, the system (2.34) is off-shell: it forms an infinite set of constraints which express all highest tensors in terms of derivatives of $C$ according to (2.36).

The above consideration is simplified by means of introducing the auxiliary coordinate $y^{n}$ and the generating function

$$
C(y \mid x)=\sum_{n=0}^{\infty} \frac{1}{n !} C_{m_{1} \ldots m_{n}}(x) y^{m_{1}} \ldots y^{m_{n}}
$$

with the convention that $C(0 \mid x)=C(x)$. The equations (2.34) then acquire the simple form

$$
\frac{\partial}{\partial x^{\underline{m}}} C(y \mid x)=\delta_{\underline{m}}^{m} \frac{\partial}{\partial y^{m}} C(y \mid x) .
$$

From this realization one concludes that the translation generators in the infinite-dimensional module $V$ of the Poincaré algebra are realized as translations in the $y$-space, i.e. $P_{n}=-\frac{\partial}{\partial y^{n}}$. The equation (2.37) reads as a covariant constancy condition

$$
d C(y \mid x)+e_{0}^{n} P_{n} C(y \mid x)=0 .
$$

Comparing this formula with (2.21) and (2.22) we see that the operator $-\sigma_{-}$is the de Rham differential in the $y$ space

$$
\sigma_{-}=-d x^{n} \frac{\partial}{\partial y^{n}} .
$$

The grading operator

$$
G=y^{n} \frac{\partial}{\partial y^{n}}, \quad\left[G, \sigma_{-}\right]=-\sigma_{-}
$$


counts a degree of the variables $y^{n}$, that is a rank $n$ of a tensor $C_{m_{1} \ldots m_{n}}$. By the Poincarè lemma we see that $H^{0}\left(\sigma_{-}, V\right)$ is one-dimensional that corresponds to a single dynamical field $C(x)$, and $H^{1}\left(\sigma_{-}, V\right)=0$ that corresponds to the absence of dynamical equations on $C(x)$.

To put the system on-shell by imposing massless or massive Klein-Gordon equation in flat Minkowski space

$$
\left(\square_{x}+m^{2}\right) C(x)=0, \quad \square_{x}=\frac{\partial^{2}}{\partial x^{m} \partial x_{m}}
$$

is equivalent to imposing the on-shell condition in the fiber variables

$$
\left(\square_{y}+m^{2}\right) C(y \mid x)=0, \quad \square_{y} \equiv \frac{\partial^{2}}{\partial y^{m} \partial y_{m}} .
$$

In terms of component tensors $C_{m_{1} \ldots m_{n}}(x)$ the latter conditions are equivalent to certain tracelessness conditions which express first traces of the fields in terms of the same set of fields. In the massless case $m=0$, the condition is [17] that the $C_{m_{1} \ldots m_{n}}(x)$ is traceless ${ }^{3}$. Indeed from (2.35) it follows that the tracelessness of $C_{n m}$ implies the Klein-Gordon equation. We refer the reader to [6] to see how the Klein-Gordon equation results from the $\sigma_{-}$cohomology $H^{1}\left(\sigma_{-}, \hat{V}\right)$ in the space $\hat{V}$ of traceless tensors.

Note that in the $1 d$ case, where indices take only one value, the set of traceless tensors $C_{m_{1} \ldots m_{n}}$ contains only two nonzero components: those with $n=0$ and $n=1$. These are the coordinate and momentum of the standard first-order (e.g., Hamiltonian) formalism.

Now we discuss invariant functionals in the scalar field case. Let us start with the off-shell case. Consider

$$
\mathcal{L}=e^{n_{1}} \wedge \ldots \wedge e^{n_{d}} \epsilon_{n_{1} \ldots n_{d}} \ell\left(C, C^{n}, C^{n m}, \ldots\right)
$$

where $\epsilon_{n_{1} \ldots n_{d}}$ is completely antisymmetric tensor and $\ell\left(C, C^{n}, C^{n m}, \ldots\right)$ is an arbitrary Lorentz invariant function (i.e., all vector indices are contracted by the Minkowski metric). The differential $Q$ acts as follows

$$
\begin{aligned}
& Q C(y \mid x)=\left(\omega_{n m} y^{n} \frac{\partial}{\partial y_{m}}+e^{m} \frac{\partial}{\partial y_{m}}\right) C(y \mid x), \\
& Q e^{n}=\omega^{n m} \wedge e_{m}, \quad Q \omega^{n m}=\omega^{n k} \wedge \omega_{k}{ }^{m},
\end{aligned}
$$

\footnotetext{
${ }^{3}$ Note that, as shown in [23] for the $3 d$ example and then in [18] for the general case, even in the massive case, one can still work with traceless tensors $C_{m_{1} \ldots m_{n}}(x)$ by modifying appropriately the form of the operator $P_{n}$ in (2.38).
} 
where the 1-forms $\omega_{n m}$ and $e^{m}$ describe Lorentz connection and frame field in any coordinates. We see that $Q \mathcal{L}=0$ because (i) $\ell\left(C, C^{n}, C^{n m}, \ldots\right)$ is Lorentz invariant (which means that all terms with $\omega_{n m}$ cancel out) and (ii)

$$
e^{n_{1}} \wedge \ldots \wedge e^{n_{d}} \wedge e^{m} \equiv 0
$$

because antisymmetrization over $d+1$ indices $n$, which take $d$ values, gives zero.

The formula (2.42) gives a most general Lorentz invariant Lagrangian of a scalar field. ( $Q$-cohomology describes actions because $Q$-exact Lagrangians are total derivatives by the unfolded equation (2.7).) Let us note that we arrived at Poincarè invariant Lagrangians because the 1-forms in the model are the gauge fields of the Poincarè algebra. Actually, from the general consideration of subsection 2.3 it follows that the constructed action is invariant under localized Poincare gauge transformations. As explained in more detail e.g. in [6], assuming that the gravitational fields $\omega^{n m}$ and $e^{n}$ are some fixed background fields this local symmetry reduces to the global Poincare symmetry (in any chosen coordinate system).

Let us now discuss conserved charges for the on-shell scalar field. We set

$$
\mathcal{L}=\epsilon_{n_{1} \ldots n_{d}} e^{n_{1}} \wedge \ldots \wedge e^{n_{d-1}} J^{n_{d}}\left(C, C^{n}, C^{n m}, \ldots\right)
$$

where $J^{k}(C)$ is a Lorentz vector. Using that

$$
\epsilon_{n_{1} \ldots n_{d-1} k} e^{n_{1}} \wedge \ldots \wedge e^{n_{d-1}} \wedge e^{m}=d^{-1} \epsilon_{n_{1} \ldots n_{d}} \delta_{k}^{m} e^{n_{1}} \wedge \ldots \wedge e^{n_{d}}
$$

we obtain

$$
Q \mathcal{L}=(-1)^{d} d^{-1} e^{n_{1}} \wedge \ldots \wedge e^{n_{d}} \epsilon_{n_{1} \ldots n_{d}} \sum_{k=1}^{\infty} C_{m_{1} \ldots m_{k}} \frac{\delta J^{m_{k}}}{\delta C_{m_{1} \ldots m_{k-1}}} .
$$

For example, taking into account that $C_{n}{ }^{n}=0$, the condition $Q \mathcal{L}=0$ is satisfied by the standard spin one current

$$
J_{n}^{\alpha \beta}=C_{n}^{\alpha} C^{\beta}-C_{n}^{\beta} C^{\alpha},
$$

where $\alpha$ and $\beta$ are color indices. One can analogously construct all other conserved currents with higher spins ${ }^{4}[24,25,8,26]$. The $Q$ cohomology factors

\footnotetext{
${ }^{4}$ Note that, in flat space, the currents with manifest dependence on the coordinates can also be analysed by virtue of introducing coordinates as new 0 -form variables $x^{n}$ satisfying the unfolded equations $d\left(x^{n}\right)-\omega^{n m} x_{m}=e^{n}$.
} 
out so-called improvements thus characterizing conserved charges rather than currents.

The example of unfolded scalar field is so simple that one might think the procedure is a sort of a trivial substitution of the original $x$ coordinates with the fiber $y$ coordinates. This is true to some extent at the free field level (especially in Minkowski background space-time with no $x$-dependence of the coefficients of the equations), but is not the case in less trivial situations like those with interactions. The advantage of the formalism is that in the sector of fiber variables it is always a sort of flat: in particular, indices are contracted by the flat Minkowski metric tensor. The situation here is analogous to that in the Fedosov quantization prescription [27] which reduces the nontrivial problem of quantization in a curved background to the standard problem of quantization of the flat phase space, that, of course, becomes an identity when the ambient space is flat itself. It is worth to mention that this parallelism is not accidental because, as one can easily see, the Fedosov quantization prescription provides a particular case of the general unfolding approach [3] in the dynamically empty (i.e. off-shell) situation. Note that parallelism between unfolded dynamics and Fedosov quantization was also discussed recently in [16].

\section{Off-Shell Unfolded Fields in Minkowski Space}

Both off-shell and on-shell unfolded formulation of free massless fields of all spins is by now well-known (see, e.g. [6] for a review and references). Before recalling details of the unfolded free field theory in section 4 , we formulate in this section the final results for the full nonlinear system of off-shell unfolded constraints for symmetric fields of all spins in flat background and its lower spin reductions to the Yang-Mills and Einstein theories.

To this end we introduce a 1 -form $A(p, y \mid x)=d x^{\underline{n}} A_{\underline{n}}(p, y \mid x)$ and 0 -form $F(p, y \mid x)$ which depend on the usual commuting space-time coordinates $x^{\underline{n}}$ and a pair of fiber Lorentz vectors $p_{n}$ and $y^{n}$ (recall that both base indices $\underline{m}, \underline{n} \ldots$ and fiber Lorentz indices $m, n \ldots$ run from 0 to $d-1)$. The variables $p_{n}$ and $y^{n}$ form a canonical pair with nonzero commutation relations

$$
\left[p_{m}, y^{n}\right]_{*}=\hbar \delta_{m}^{n}
$$

where $[a, b]_{*}=a * b-b * a$, the deformation "Planck constant" parameter $\hbar$ is introduced for the future convenience and the Weyl star product is defined in 
the standard way

$$
(f * g)(p, y)=f(p, y) \exp \frac{\hbar}{2}\left(\frac{\overleftarrow{\partial}}{\partial p^{n}} \frac{\partial}{\partial y_{n}}-\frac{\overleftarrow{\partial}}{\partial y^{n}} \frac{\partial}{\partial p_{n}}\right) g(p, y)
$$

Let $D$ be the covariant derivative

$$
D=\hbar d+A=d x^{\underline{n}}\left(\hbar \frac{\partial}{\partial x^{\underline{n}}}+A_{\underline{n}}(p, y \mid x)\right) .
$$

The nonlinear unfolded off-shell HS formulation in flat space takes extremely simple form of the zero-curvature and covariant constancy conditions

$$
D^{2}=0, \quad D(F)=0
$$

that is

$$
d A+\hbar^{-1} A * A=0, \quad d F+\hbar^{-1}[A, F]_{*}=0 .
$$

In addition there is a "boundary condition" that

$$
A=A_{0}+A_{1}, \quad F=F_{0}+F_{1},
$$

where

$$
A_{0}=d x^{\underline{n}} e_{\underline{\underline{n}}}^{n} p_{n}+\omega_{\underline{\underline{n}}}^{n m} p_{n} y_{m}
$$

and

$$
F_{0}=\frac{1}{2} p_{n} p^{n}
$$

are zero-order parts while $A_{1}$ and $F_{1}$ are fluctuations. Here $e_{\underline{n}}^{n}$ and $\omega_{\underline{n}}^{n m}=$ $-\omega_{\underline{n}}{ }^{m n}$ describe frame 1-form and Lorentz connection of the background Minkowski space-time, i.e. $e_{\underline{n}}^{n}(x)$ is a nondegenerate matrix and the vacuum equation

$$
d A_{0}+\hbar^{-1} A_{0} * A_{0}=0
$$

is satisfied, which in turn is equivalent to the zero-torsion condition (2.13) (the part proportional to $p^{n}$ ) and vanishing Riemann tensor condition (2.14) (the part proportional to $\left.p^{[n} y^{m]}\right)$. A solution that describes Minkowski space-time in Cartesian coordinates is $e_{\underline{n}}^{m}=\delta_{\underline{n}}^{m}$ and $\omega_{\underline{n}}{ }^{m n}=0$.

The fields $A(p, y \mid x)$ and $\bar{F}(p, y \mid \bar{x})$ are power series in the fiber variables $p_{n}$ and $y^{m}$. It is convenient to expand them in powers of the fiber momenta $p_{n}$

$$
A(p, y \mid x)=\sum_{s=1}^{\infty} d x^{\underline{n}} A_{\underline{n}}^{n_{1} \ldots n_{s-1}}(y \mid x) p_{n_{1}} \ldots p_{n_{s-1}},
$$




$$
F(p, y \mid x)=\sum_{s=0}^{\infty} F^{n_{1} \ldots n_{s}}(y \mid x) p_{n_{1}} \ldots p_{n_{s}} .
$$

A massless spin $s$ field is described by the 1-forms $A(p, y \mid x)$ and 0 -forms $F(p, y \mid x)$ which are, respectively, of order $s-1$ and $s$ in $p$.

The equations (3.4) are invariant under the gauge transformations

$$
\delta A=D \epsilon=d \epsilon+\hbar^{-1}[A, \epsilon]_{*}, \quad \delta F=\hbar^{-1}[F, \epsilon]_{*},
$$

where $\epsilon(p, y \mid x)$ is an arbitrary function of its arguments. As will be shown in section 6 , some of these gauge parameters are responsible for gauging away redundant degrees of freedom while the rest become the usual lower spin and higher spin gauge symmetry parameters (like, e.g., standard Yang-Mills symmetry).

A global HS symmetry of the vacuum solution (3.7) and (3.8) has parameters $\epsilon(p, y)$ that commute to $\frac{1}{2} p^{2}$. As shown in section 6 , this means that they are described by the polynomials

$$
\epsilon(p, y)=\epsilon^{n_{1} \ldots n_{s-1}, m_{1} \ldots m_{t}} p_{n_{1}} \ldots p_{n_{s-1}} y_{m_{1}} \ldots y_{m_{t}}
$$

where the parameters $\epsilon^{n_{1} \ldots n_{s-1}, m_{1} \ldots m_{t}}$ have the symmetry properties of Young tableaux with $s-1$ cells in the upper row and $t$ cells in the second one. This set of symmetries precisely matches that of the HS algebra of [2]. The two HS algebras are not isomorphic, however. The algebra considered in this paper is a contraction of the (off-shell) $A d S$ HS algebra of [2] pretty much like Poincarè algebra is a contraction of the $A d S$ algebra.

The system (3.4) is obviously consistent and nonlinear. Despite it has the trivial form of zero-curvature and covariant constancy conditions ${ }^{5}$, it describes nontrivial models including gravity and Yang-Mills. This is possible because the 0 -form $F$ has a nonzero vacuum expectation value $F_{0}(3.8)$ so that the gauge symmetries are spontaneously broken and the flat 1-form connection $A(p, y \mid x)$ cannot be completely gauged away by the leftover unbroken gauge symmetries. To prove that the system (3.4) describes appropriately off-shell relativistic fields it is enough to check that it is doing so at the linearized level. This will be shown in section 6 .

The off-shell HS system (3.4) admits simple truncations to the lower spin sectors of spins zero, one and two. This may sound surprising because it is

\footnotetext{
${ }^{5}$ Let us note that the form of the system (3.4) is analogous to that of the basis system of the Fedosov quantization prescription [27]. The difference is that we use its restriction to the Lagrangian base subsurface $M^{d}$ of the corresponding noncommutative $2 d$-dimensional phase space. Analogous system was also considered in [28] in a different context.
} 
usually claimed that lower-spin fields are sources to the HS fields (see e.g. [8]). Analogously to the argument on the (ir)relevance of the $A d S$ background, although this is indeed true on-shell, it may not be so off-shell where no equations of motion are imposed on the dynamical fields and, therefore, no obstruction results from a nontrivial $\sigma_{-}$cohomology associated with the equations of motion to impose restrictions on spin spectra and/or geometry of the background space-time. In other words, as long as there are no nontrivial dynamical equations on dynamical fields, there is also no question on how their right hand sides (currents) are built of other fields. In the absence of dynamical field equations, i.e. when all relations are some constraints, whatever is added to the right hand sides of the constraints it can always be absorbed into a redefinition of auxiliary fields.

In the case of gravity, the connection 1-form $A(p, y \mid x)$ is linear in $p^{n}$

$$
A(p, y \mid x)=A^{n}(y \mid x) p_{n} .
$$

Because every commutator takes away at least one power of $p^{n}$, the left hand side of the zero curvature equation in (3.4) is also linear in $p^{n}$. With the help of the star product (3.2) one obtains

$$
d A^{n}(y \mid x)+A^{m}(y \mid x) \wedge \frac{\partial}{\partial y^{m}} A^{n}(y \mid x)=0 .
$$

This is the zero curvature equation for the Lie algebra of vector fields in the variables $y^{n}$.

The spin two 0 -form $F(p, y \mid x)$ is bilinear in $p^{n}$. Let us also introduce a spin zero (i.e., $p^{n}$-independent) "dilaton" field by setting

$$
F(p, y \mid x)=F^{m n}(y \mid x) p_{m} p_{n}+F(y \mid x) .
$$

The covariant constancy condition on $F$ in (3.4) amounts to

$$
\begin{aligned}
d F^{m n}(y \mid x) & +A^{l}(y \mid x) \frac{\partial}{\partial y^{l}} F^{m n}(y \mid x) \\
& -\frac{\partial}{\partial y^{l}} A^{m}(y \mid x) F^{l n}(y \mid x)-\frac{\partial}{\partial y^{l}} A^{n}(y \mid x) F^{l m}(y \mid x)=0
\end{aligned}
$$

and

$$
d F(y \mid x)+A^{l}(y \mid x) \frac{\partial}{\partial y^{l}} F(y \mid x)+\frac{\hbar^{2}}{4} \frac{\partial^{2}}{\partial y^{l} \partial y^{n}} A^{m}(y \mid x) \frac{\partial}{\partial y^{m}} F^{l n}(y \mid x)=0 .
$$


The equation (3.16) is the covariant constancy condition for the second rank symmetric tensor $F^{m n}(y \mid x)$. The equation (3.17) contains two types of terms. The $\hbar$-independent terms represent the vector field covariant constancy condition in the scalar representation. The $\hbar-$ dependent term describes a source term built of the gravitational fields. Since the system remains consistent for any value of $\hbar$, one can consistently set $\hbar$ equal to zero. This limit corresponds to the Poisson bracket realization of the algebra of vector fields, where the equation (3.17) just describes a scalar field in the external gravitational field.

That the last term in the equation (3.17) can be added is very interesting, however. It describes a nontrivial co-cycle of the algebra of vector fields with polynomial coefficients. Having three derivatives, it is somewhat reminiscent of the central extension of the Witt algebra to Virasoro.

The system is invariant under the $y$-vector field transformations ("diffeomorphisms" with the coefficients polynomial in $y$ ) gauged in $x$ space-time

$$
\begin{gathered}
\delta A^{n}(y \mid x)=d \epsilon^{n}(y \mid x)+A^{m}(y \mid x) \frac{\partial}{\partial y^{m}} \epsilon^{n}(y \mid x)-\epsilon^{m}(y \mid x) \frac{\partial}{\partial y^{m}} A^{n}(y \mid x), \\
\delta F^{m n}(y \mid x)=\frac{\partial}{\partial y^{l}} \epsilon^{m}(y \mid x) F^{l n}(y \mid x)+\frac{\partial}{\partial y^{l}} \epsilon^{n}(y \mid x) F^{l m}(y \mid x)-\epsilon^{l}(y \mid x) \frac{\partial}{\partial y^{l}} F^{m n}(y \mid x), \\
\delta F(y \mid x)=-\epsilon^{l}(y \mid x) \frac{\partial}{\partial y^{l}} F(y \mid x)-\frac{\hbar^{2}}{4} \frac{\partial^{2}}{\partial y^{l} \partial y^{n}} \epsilon^{m}(y \mid x) \frac{\partial}{\partial y^{m}} F^{l n}(y \mid x)=0,
\end{gathered}
$$

where $\epsilon^{l}(y \mid x)$ is an arbitrary function of $x^{\underline{n}}$ and $y^{n}$ that expands in power series of $y^{n}$.

Let us note that if $A(p, y \mid x)$ and $F(p, y \mid x)$ were matrices (i.e. took values in some noncommutative associative algebra), the property that every commutator takes away at least one power of $p^{n}$ would not be true any more $e^{6}$. This means that if colored spin two particles appear, this is only possible in the presence of HS fields, which conclusion is in agreement with the results of [29].

As explained in sections 6 and 7, the equations (3.14) and (3.16) provide unfolded formulation of off-shell nonlinear gravity. The equation (3.17) with $\hbar \neq 0$ extends it in a non-trivial way to a system with dilaton. It can be further extended to a system of fields with spins $s \leq 1$.

\footnotetext{
${ }^{6}$ Indeed, a wedge product of matrix valued connection 1-form $A^{i}{ }_{j}(p)$ linear in $p$ will generically contain terms bilinear in $p^{n}$ because $[a \otimes x, b \otimes y]=\frac{1}{2}([a, b] \otimes\{x, y\}+\{a, b\} \otimes$ $[x, y])$, for $a, b \in A_{1}$ and $x, y \in A_{2}$ where $A_{1,2}$ are two associative algebras, i.e. when the matrix commutator is not zero, the star product anticommutator of the functions of $y$ and $p$ appears.
} 
A spin one massless field is described by the 1-form

$$
\mathcal{A}=\hbar^{-1} d x^{\underline{n}} \mathcal{A}_{\underline{n}}(y \mid x)
$$

along with the 0 -form $p_{n} F^{n}(y \mid x)$, both taking values in a matrix algebra of a non-Abelian Yang-Mills theory. Spin zero matter fields are described by a 0-form $F(y \mid x)$ in some representation of the Yang-Mills algebra. The corresponding equations read as

$$
\begin{aligned}
d \mathcal{A}(y \mid x)+A^{m}(y \mid x) & \wedge \frac{\partial}{\partial y^{m}} \mathcal{A}(y \mid x)+\mathcal{A}(y \mid x) \wedge \mathcal{A}(y \mid x)=0 \\
d F^{m}(y \mid x)-\frac{\partial}{\partial y_{m}} \mathcal{A}(y \mid x) & +A^{l}(y \mid x) \frac{\partial}{\partial y^{l}} F^{m}(y \mid x)-\frac{\partial}{\partial y^{n}} A^{m}(y \mid x) F^{n}(y \mid x) \\
& +\left[\mathcal{A}(y \mid x), F^{m}(y \mid x)\right]=0
\end{aligned}
$$

and

$$
d F(y \mid x)+A^{l}(y \mid x) \frac{\partial}{\partial y^{l}} F(y \mid x)+\mathcal{A}(y \mid x)(F(y \mid x))=0,
$$

where $A^{l}(y \mid x)$ describes the background gravitational field, [, ] is the usual commutator in the matrix algebra where the Yang-Mills fields $\mathcal{A}(y \mid x)$ and $F^{n}(y \mid x)$ take their values while $\mathcal{A}(y \mid x)(F(y \mid x))$ is a result of the action of the YangMills field $\mathcal{A}(y \mid x)$ on the scalar field $F(y \mid x)$ according to the representation of the Yang-Mills algebra carried by $F(y \mid x)$.

The corresponding gauge transformation law is

$$
\begin{aligned}
& \delta \mathcal{A}(y \mid x)=d \epsilon(y \mid x)+A^{m} \frac{\partial}{\partial y^{m}} \epsilon(y \mid x)-\epsilon^{m}(y \mid x) \frac{\partial}{\partial y^{m}} \mathcal{A}(y \mid x)+[\mathcal{A}(y \mid x), \epsilon(y \mid x)], \\
& \delta F^{m}(y \mid x)=\frac{\partial}{\partial y_{m}} \epsilon(y \mid x)-\epsilon^{l}(y \mid x) \frac{\partial}{\partial y^{l}} F^{m}(y \mid x)+\frac{\partial}{\partial y^{n}} \epsilon^{m}(y \mid x) F^{n}(y \mid x) \\
&+ {\left[F^{m}(y \mid x), \epsilon(y \mid x)\right]=0, }
\end{aligned}
$$

and

$$
\delta F(y \mid x)=-\epsilon^{l}(y \mid x) \frac{\partial}{\partial y^{l}} F(y \mid x)-\epsilon(y \mid x)(F(y \mid x))=0 .
$$

It forms a semidirect product of the Yang-Mills algebra with $y$-dependent parameters $\epsilon(y \mid x)$ (forming an ideal) and the algebra of $y$-space vector fields $\epsilon^{m}(y \mid x)$ polynomial in $y$. 
Let us note that the unfolded formulation of Yang-Mills theory and gravity presented here is deeply related to earlier important work on a reformulation of these fundamental theories. In particular, relevance of the $y$-dependent symmetries to the description of Yang-Mills theory was originally found in the sigma-model approach of Ivanov and Ogievetsky [30]. The idea that twistor transform of the full nonlinear Yang-Mills equations can be achieved via doubling of space-time coordinates in the Yang-Mills connection was put forward by Witten [31] (see also [32]). Ivanov observed [33] that Cartan forms built of the goldstonion fields of the sigma-model approach to Yang-Mills theory satisfy the zero-curvature equation (3.22). Analogous construction for the case of gravity was developed by Pashnev in [34], where it was shown that the relevant infinite-dimensional algebra is the algebra of $y$-space diffeomorphisms while the Cartan 1-forms, built of the goldstonion fields of the sigma-model approach to gravity, satisfy the zero-curvature equation (3.14). Note that, from the perspective of our approach, the goldstonion fields of $[30,33,34]$ appear in the exponential parametrization of the gauge function $g(y \mid x)$ of the pure gauge representation $A=g^{-1} d g$ for solutions of the zero curvature equations (3.22) or (3.14).

As will be explained elsewhere, it is also possible to include spin $1 / 2$ matter fields into our lower-spin model. On the other hand, if any (boson or fermion) HS field with $s>2$ is added, the system (3.4) requires an infinite set of HS fields to be included. This is so because the commutator of the connection $A(p, y \mid x)$ of order $s_{1}-1$ in $p$ with the 0 -form $F(p, y \mid x)$ of order $s_{2}$ in $p$ contributes to the part of order $s_{1}+s_{2}-2$ in $p$ in the equation (3.4) for $F$. This means that spin $s_{1}$ and spin $s_{2}$ fields produce a source for spin $s=s_{1}+s_{2}-2$. As a result, any spin greater than two gives rise to an infinite tower of higher and higher spins. In other words if a HS field is present in the system under consideration, no truncation to a subsystem with a finite number of spins is available. It is possible, however, to truncate the system to only even spins where every even spin appears just once.

The property that HS fields form infinite towers is a consequence of the structure of the star product algebra. On the other hand, since unfolding of only Bianchi identities with no dynamical equations imposed is always possible, it should be possible to formulate an off-shell HS system for any given spin in the background gravitational and/or Yang-Mills fields. As discussed in more detail in section 9 , this is achieved by replacing the star product algebra with the commutative algebra of functions of commuting variables $y^{n}$ and $p_{n}$ in the spin $s \neq 2$ sector and with the Poisson algebra in the spin two sector of connections linear in $p$. 


\section{Free Fields Unfolded}

To explain how the equations (3.4) describe a nonlinear HS system we recall in this section the unfolded formulation of free totally symmetric massless fields of any spin elaborated in [35] for the $d=4$ case and then extended to any dimension in [36, 37]. We first consider important examples of spin one in subsection 4.1 and spin two in subsection 4.2, coming to the general case in subsection 4.3.

\subsection{Free spin one}

A spin one gauge field is described by a vector potential $a_{\underline{n}}(x)$. One writes

$$
d a=e_{0}^{n} \wedge e_{0}^{m} C_{n, m},
$$

where $e_{0}^{n}=d x^{n}$ is the frame 1 -form of the background Minkowski space and the antisymmetric tensor $C_{n, m}=-C_{m, n}$ parametrizes components of the field strength. It is depicted by the Young tableau $B$. The first derivative of the field strength

$$
d C_{n, m}=e_{0}^{p} C_{n, m ; p}
$$

has the structure

$$
\mathrm{日} \otimes \mathrm{a}=\text { 目 } \oplus \text { 巴 } .
$$

The Bianchi identities for (4.1) imply however that the part that contains antisymmetrization over three indices in (4.2) must be zero: $\mathrm{\theta}=0$. This means that

$$
d C_{n, m}=e_{0}^{p}\left(C_{n p, m}-C_{m p, n}\right),
$$

where $C_{n p, m}$ has symmetry properties of the hook Young tableau being otherwise arbitrary, i.e. $C_{m n, p}=C_{n m, p}$ and symmetrization over three indices gives zero: $C_{n m, p}+C_{n p, m}+C_{m p, n}=0$. The equation (4.4) is the first step of the unfolding of spin one dynamics in the 0 -form sector. The next step is to analyse Bianchi identities for (4.4) that impose restrictions on the first derivative of $C_{n p, m}$. The process continues indefinitely leading to the following chain of differential relations

$$
d C_{m_{1} \ldots m_{l}, n}=e_{0}^{p}\left((l+1) C_{m_{1} \ldots m_{l} p, n}+C_{m_{1} \ldots m_{l} n, p}\right)
$$

with the 0-forms $C_{m_{1} \ldots m_{l}, n}$ described by the Young tableau $C_{m_{1} \ldots m_{l}, n}$ is symmetric in the indices $m$ and such that symmetrization over all 
$l+1$ indices gives zero

$$
C_{\left\{m_{1} \ldots m_{l}, m_{l+1}\right\}}=0 .
$$

The system (4.1) and (4.5) gives off-shell unfolded formulation of spin one field because in its derivation we did not make use of the Maxwell equations. The latter require the first derivative of the Maxwell tensor to be traceless. In other words, Maxwell equations require the tensor $C_{m n, k}$ on the right hand side of (4.4) to be traceless. From (4.5) it follows then that all tensors $C_{m_{1} \ldots m_{l}, n}$ are also traceless

$$
C_{m_{1} \ldots m_{l}, n} \eta^{m_{1} m_{2}}=0, \quad C_{m_{1} \ldots m_{l}, n} \eta^{m_{1} n}=0 .
$$

Therefore, the system (4.1) and (4.5) along with the conditions (4.6), (4.7) gives unfolded formulation of the Maxwell equations, i.e., the on-shell unfolded formulation of a spin one free massless particle.

The traceful (traceless) 0 -forms $C_{m_{1} \ldots m_{l}, n}$ form a basis of the space of all gauge invariant derivatives in the off-shell (on-shell) Maxwell theory. They can be described by a generating function

$$
C(p, y \mid x)=\sum_{l=1}^{\infty} C_{m_{1} \ldots m_{l}, n} y^{m_{1}} \ldots y^{m_{l}} p^{n}
$$

satisfying the Young condition

$$
y^{n} \frac{\partial}{\partial p^{n}} C(p, y \mid x)=0
$$

equivalent to (4.6). To put the free spin one system on-shell is equivalent to imposing the tracelessness conditions

$$
\frac{\partial^{2}}{\partial y^{m} \partial y_{m}} C(p, y \mid x)=0, \quad \frac{\partial^{2}}{\partial y^{m} \partial p_{m}} C(p, y \mid x)=0 .
$$

Suppose now that we want to reformulate analogously a non-Abelian YangMills theory. To this end one replaces the left hand side of (4.1) by the nonAbelian Yang-Mills field strength

$$
d a+\frac{1}{2}[a, a]=e_{0}^{n} \wedge e_{0}^{m} C_{n, m},
$$

where both $a$ and $C_{n, m}$ take values in a Yang-Mills Lie algebra. The Bianchi identities then give a relation analogous to (4.4)

$$
D C_{n, m}=e_{0}^{p}\left(C_{n p, m}-C_{m p, n}\right),
$$


where $D$ is the Yang-Mills covariant derivative in the adjoint representation. The analysis of the Bianchi identity for this equation is more complicated because $D^{2}$ is itself proportional to $C_{n, m}$ by virtue of (4.11). As a result, the equations (4.5) with higher $l$ must acquire nonlinear corrections. To the best of our knowledge a full nonlinear form of the unfolded spin one system was not known so far both in the off-shell and in the on-shell cases. One of the results of this paper consists of the observation that the equations (3.4) solve the off-shell Yang-Mills problem when restricted to the spin one case. We will also show in section 8 how the nonlinear Yang-Mills equations look like in these terms.

\subsection{Linearized gravity}

The set of fields in Einstein-Cartan's formulation of gravity consists of the frame field $e_{\underline{m}}^{m}$ and the Lorentz connection $\omega_{\underline{m}}^{n m}$. One supposes that the torsion constraint (2.13) is satisfied in order to express the Lorentz connection in terms of the frame field. The Lorentz curvature can be expressed as $R^{m n}=$ $e_{k} \wedge e_{l} R^{m n ; k l}$, where $R^{m n ; k l}$ is a rank four tensor antisymmetric in the pair of indices $m n$ and $k l$, having the symmetries of the tensor product $\theta \otimes 日$. The algebraic Bianchi identity $e_{n} \wedge R^{m n}=0$, which follows from the zero torsion constraint, implies that the tensor $R^{m n ; k l}$ possesses the symmetries of the Riemann tensor, i.e. $R^{[m n ; k] l}=0$. This means that it carries an irreducible representation of $G L(d)$ characterized by the Young tableau $\boxplus$.

For our purpose, it is more convenient to use the symmetric basis. In this convention, one supplements the zero torsion condition (2.13) with the equation

$$
R^{m n}=e_{k} \wedge e_{l} C^{m k, n l},
$$

where the 0-form $C^{m k, n l}$ is symmetric in the pairs $m k$ and $n l$ and satisfies the algebraic relation $C^{\{m k, n\} l}=0$, which implies the window Young symmetry $\boxplus$ in the symmetric basis. To start the unfolding of linearized gravity around the Minkowski background one linearizes the equation of (4.13) to

$$
R_{1}^{m n}=e_{0 k} \wedge e_{0 l} C^{m k, n l} .
$$

To unfold this equation one has to add the equations containing the differential of the Riemann 0-form $C^{m k, n l}$. Since we do not want to impose any additional dynamical restrictions on the system, the only restrictions on the derivatives

of the Riemann 0-form $C^{m k, n l}$ result from the Bianchi identities applied to (4.14). 
A priori, the first Lorentz covariant derivative of the Riemann tensor is a rank five tensor in the following representation

$$
\boxplus \otimes \square=\boxplus \oplus \boxplus
$$

of $g l(d)$. The Bianchi identity applied to (4.14) implies that the part of the derivative of the Riemann tensor antisymmetrized in three indices must vanish. As a result, in the decomposition (4.15) of the Lorentz covariant derivative of the Riemann tensor, the first term vanishes and the second term is arbitrary. This is equivalent to say that

$$
d C^{m k, n l}=e_{0 f}\left(2 C^{m k f, n l}+C^{m k n, l f}+C^{m k l, n f}\right),
$$

where the right hand side is fixed by the Young symmetry properties of the left hand side modulo an overall normalization coefficient. This equation is the first step of the unfolding procedure in the sector of 0 -forms. $C^{m k f, n l}$ is irreducible under $g l(d)$. The analysis of the Bianchi identities goes on indefinitely resulting in the infinite set of equations

$d C^{m_{1} \ldots m_{k+2}, n_{1} n_{2}}=e_{0 l}\left((k+2) C^{m_{1} \ldots m_{k+2} l, n_{1} n_{2}}+C^{m_{1} \ldots m_{k+2} n_{1}, n_{2} l}+C^{m_{1} \ldots m_{k+2} n_{2}, n_{1} l}\right)$

$(0 \leq k<\infty)$, where the fields $C^{m_{1} \ldots m_{k+2}, n_{1} n_{2}}$ are described by the two-row Young tableau i.e.

$$
C^{\left\{m_{1} \ldots m_{k+2}, n_{1}\right\} n_{2}}=0 .
$$

As expected, the system (4.17) is consistent with $d^{2} C^{m_{1} \ldots m_{k+2}, n_{1} n_{2}}=0$.

Analogously to the spin zero and spin one cases, the meaning of the 0 -forms $C^{m_{1} \ldots m_{k+2}, n_{1} n_{2}}$ is that they form a basis in the space of all gauge invariant combinations of the derivatives of the spin two gauge field. The set of 0 -forms $C^{m_{1} \ldots m_{k+2}, n_{1} n_{2}}$ can be conveniently described by a generating function

$$
C(p, y \mid x)=\sum_{l=2}^{\infty} C_{m_{1} \ldots m_{l}, n_{1} n_{2}} y^{m_{1}} \ldots y^{m_{l}} p^{n_{1}} p^{n_{2}},
$$

satisfying the Young condition that has the same form (4.9) as in the spin one case.

The vacuum Einstein equations which put the system on-shell state that $C_{m n, k l}$ is traceless. In other words, the Riemann tensor is equal on-shell to the Weyl tensor. By virtue of Bianchi identities, at the linearized level, all derivatives of the Weyl tensor are also traceless on-shell. (For more detail see, e.g., 
[6]). In other words, unfolded formulation of the linearized spin two equations is given by the equations (4.14) and (4.17) at the condition that the fields $C^{m_{1} \ldots m_{k+2}, n_{1} n_{2}}$ satisfy (4.18) and are all traceless, $\eta_{m_{1} m_{2}} C^{m_{1} m_{2} \ldots m_{k+2}, n_{1} n_{2}}=0$, which condition has the same form (4.10) as in the spin one case. The traceless 0 -forms $C^{m_{1} \ldots m_{k+2}, n_{1} n_{2}}$ form a basis in the space of all on-mass-shell nontrivial gauge invariant combinations of the derivatives of the spin two gauge field.

To extend the free field unfolded formulation of the massless spin two field to the nonlinear level one has to replace $d$ and $e_{0}^{n}$ with the Lorentz covariant derivative $D^{L}$ and the dynamical frame field $e^{n}$, respectively. Since $D^{L} D^{L}$ is the Riemann tensor, Bianchi identities for the covariantized equations (4.17) will require nonlinear corrections to these equations analogous to those in the Yang-Mills theory. The nonlinear corrections to the covariantized field equations (4.17) quadratic in the 0 -forms $C$ were found in [4] for the $\mathrm{d}=4$ case. In this paper we find both off-shell and on-shell nonlinear formulation for spin two in any dimension.

\subsection{Free symmetric massless fields of any spin}

Free unfolded HS field equations can be formulated [35, 36, 37] in terms of the 1-form gauge fields which generalize those of the Cartan formulation of gravity. Namely, for a massless spin $s$, one introduces a set of 1-forms $d x \underline{\underline{m}} \omega_{\underline{m}}^{n_{1} \ldots n_{s-1}, m_{1} \ldots m_{t}}$ which have the symmetry of the two-row Young tableaux

with $s-1$ cells in the first row and any $0 \leq t \leq s-1$ cells in the second row with respect to the fiber indices $n$ and $m$.

In the on-shell system considered in [35, 36, 37], the connections $d x \underline{\underline{m}} \omega_{m}^{n_{1} \ldots n_{s-1}, m_{1} \ldots m_{t}}$ are traceless in the fiber indices $n$ and $m$. As shown in $[5,6]$, the off-shell version is obtained by relaxing the tracelessness condition. We first discuss the off-shell case with traceful connections.

The frame-like HS field is that with $t=0$

$$
d x^{\underline{m}} e_{\underline{m}}^{n_{1} \ldots n_{s-1}}=d x \underline{\underline{m}} \omega_{\underline{m}}^{n_{1} \ldots n_{s-1}} .
$$

The Lorentz connection-like auxiliary field has $t=1$, i.e. $\omega_{m}^{n_{1} \ldots n_{s-1}, m}$. The fields with $t>1$ are called extra fields. They appear for higher spins with $s>2$. The set of gauge fields $\omega_{\underline{m}}^{n_{1} \ldots n_{s-1}, m_{1} \ldots m_{t}}$ generalizes the sets of spin one and spin two gauge fields to any spin, containing them as particular cases. The flat space linearized HS curvatures have the form

$$
R_{1}^{n_{1} \ldots n_{s-1}, m_{1} \ldots m_{t}}=D_{0}^{L} \omega^{n_{1} \ldots n_{s-1}, m_{1} \ldots m_{t}}+e_{0 k} \omega^{n_{1} \ldots n_{s-1}, m_{1} \ldots m_{t} k},
$$


where $D_{0}^{L}$ and $e_{0}^{n}$ are, respectively, the Lorentz covariant differential and the frame 1 -form of the background Minkowski space $\left(D^{L}=d\right.$ and $e_{0}^{n}=d x^{n}$ in Cartesian coordinates). These linearized curvatures generalize to any spin the spin one Maxwell field strength and spin two linearized torsion $(t=0)$ and Riemann tensor $(t=1)$.

The linearized HS gauge transformations have the form

$$
\delta \omega^{n_{1} \ldots n_{s-1}, m_{1} \ldots m_{t}}=D_{0}^{L} \epsilon^{n_{1} \ldots n_{s-1}, m_{1} \ldots m_{t}}+e_{0 k} \epsilon^{n_{1} \ldots n_{s-1}, m_{1} \ldots m_{t} k},
$$

where the 0 -form $\epsilon^{n_{1} \ldots n_{s-1}, m_{1} \ldots m_{t}}(x)$ is an arbitrary gauge parameter with the symmetry properties of the two-row Young tableau with $s-1$ cells in the first row and $t$ cells in the second row.

The HS analogue of the equation (4.1) for spin one and the equation (4.14) for spin two is

$$
R_{1}^{n_{1} \ldots n_{s-1}, m_{1} \ldots m_{t}}=\delta_{t, s-1} e_{0 k} \wedge e_{0 l} C^{n_{1} \ldots n_{s-1} k, m_{1} \ldots m_{s-1} l} \quad(0 \leq t \leq s-1) .
$$

The 0-form $C^{n_{1} \ldots n_{s}, m_{1} \ldots m_{s}}$ is the spin- $s$ Riemann-like tensor. It is characterized

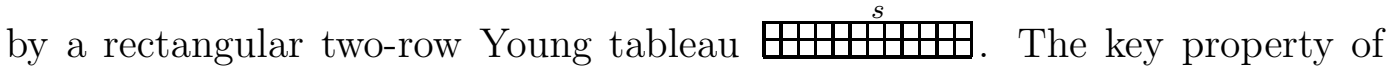
this system is [6] that it imposes no restrictions on the frame-like connection (4.20). All connections $\omega_{\underline{m}}^{n_{1} \ldots n_{s-1}, m_{1} \ldots m_{t}}$ with $t>0$ are expressed via its order$t$ derivatives modulo Lorentz-like Stueckelberg gauge symmetries (4.22) with $\epsilon^{n_{1} \ldots n_{s-1}, m_{1} \ldots m_{t}}$ with $t>0$. The Riemann tensor $C^{n_{1} \ldots n_{s}, m_{1} \ldots m_{s}}$ represents order-s gauge invariant combinations of the derivatives of the spin $s$ frame-like field and coincides with the analogous tensor found in the metric-like formalism by de Wit and Freedman [38].

The analysis of the Bianchi identities of (4.23) works for any spin $s \geq 2$ in a way analogous to gravity. The final result is the following equation which has a form of a covariant constancy condition

$$
\begin{aligned}
0= & \widetilde{D}_{0} C^{n_{1} \ldots n_{s+k}, m_{1} \ldots m_{s}} \equiv D_{0}^{L} C^{n_{1} \ldots n_{s+k}, m_{1} \ldots m_{s}} \\
& \quad-e_{0 k}\left((2+k) C^{n_{1} \ldots n_{s+k} k, m_{1} \ldots m_{s}}+s C^{n_{1} \ldots n_{s+k}\left\{m_{1}, m_{2} \ldots m_{s}\right\} k}\right)
\end{aligned}
$$

$0 \leq k<\infty$, where $C^{n_{1} \ldots n_{s+k}, m_{1} \ldots m_{s}}$ are $g l(d)$ tensors characterized by the Young tableaux mentrivial $k$-th derivatives of the spin- $s$ Riemann-like tensor, thus forming a basis in the space of gauge invariant combinations of derivatives of a spin $s$ HS gauge field. The system (4.24) is an extension of the spin zero, spin one and spin two off-shell systems considered 
above to any spin. Let us stress that, for $s \geq 1$, the infinite system of equations (4.24) describes constraints that expresses higher tensors $C^{n_{1} \ldots n_{s+k}, m_{1} \ldots m_{s}}$ in terms of derivatives of the Riemann-like tensors $C^{n_{1} \ldots n_{s}, m_{1} \ldots m_{s}}$ along with consequences of (4.23) by the Bianchi identities. Note that (4.23) makes no sense for $s=0$ because there is no spin 0 gauge potential while (4.24) with $s=0$ reproduces the unfolded spin zero off-shell equation (2.34). The fact that the system (4.23) and (4.24) imposes no differential conditions on the frame-like connection (4.20) and the spin zero 0-form $C[6]$ will be referred to as Central off-mass-shell theorem.

The set of HS connection 1-forms $\omega^{n_{1} \ldots n_{s-1}, m_{1} \ldots m_{t}}$ and Riemann 0-forms $C^{n_{1} \ldots n_{s+k}, m_{1} \ldots m_{s}}$ can be described by the generating functions

$$
\omega(p, y \mid x)=\sum_{l, k} \omega_{m_{1} \ldots m_{l}, n_{1} \ldots n_{k}} p^{m_{1}} \ldots p^{m_{l}} y^{n_{1}} \ldots y^{n_{k}}
$$

and

$$
C(p, y \mid x)=\sum_{l, k} C_{m_{1} \ldots m_{l}, n_{1} \ldots n_{k}} y^{m_{1}} \ldots y^{m_{l}} p^{n_{1}} \ldots p^{n_{k}},
$$

which satisfy the conditions

$$
\begin{aligned}
& \tau_{+} \omega(p, y \mid x)=0, \\
& \tau_{-} C(p, y \mid x)=0,
\end{aligned}
$$

where the operators

$$
\tau_{+}=p^{n} \frac{\partial}{\partial y^{n}}, \quad \tau_{-}=y^{n} \frac{\partial}{\partial p^{n}}, \quad \tau_{0}=\left[\tau_{-}, \tau_{+}\right]=y^{n} \frac{\partial}{\partial y^{n}}-p^{n} \frac{\partial}{\partial p^{n}}
$$

form a $s p(2)$ algebra. The conditions (4.27) and (4.28) imply that symmetrization over any $l+1$ indices of the coefficients $\omega_{m_{1} \ldots m_{l}, n_{1} \ldots n_{k}}$ and $\mathbf{C}_{m_{1} \ldots m_{l}, n_{1} \ldots n_{k}}$ gives zero. This is equivalent to the fact that they are described by the two-row Young tableaux with $l$ cells in the first row and $k$ cells in the second row.

For a fixed spin $s$ one has

$$
\begin{gathered}
p^{n} \frac{\partial}{\partial p^{n}} \omega(p, y \mid x)=(s-1) \omega(p, y \mid x), \\
p^{n} \frac{\partial}{\partial p^{n}} C(p, y \mid x)=s C(p, y \mid x) .
\end{gathered}
$$


Following [35, 36, 37], to put the system on-shell one requires both the HS connections $\omega(p, y \mid x)$ and 0 -forms $C(p, y \mid x)$ to be traceless, that is harmonic in the fiber variables $y$ and $p$

$$
\begin{array}{llrl}
\frac{\partial^{2}}{\partial y^{m} \partial y_{m}} \omega(p, y \mid x)=0, & \frac{\partial^{2}}{\partial y^{m} \partial p_{m}} \omega(p, y \mid x)=0, & \frac{\partial^{2}}{\partial p^{m} \partial p_{m}} \omega(p, y \mid x)=0 . \\
\frac{\partial^{2}}{\partial y^{m} \partial y_{m}} C(p, y \mid x)=0, & \frac{\partial^{2}}{\partial y^{m} \partial p_{m}} C(p, y \mid x)=0, & \frac{\partial^{2}}{\partial p^{m} \partial p_{m}} C(p, y \mid x)=0 .
\end{array}
$$

The resulting field equations (4.23) generalize (4.14) of linearized gravity. That they indeed describe free equations of motion for any spin equivalent to the equations, which follow from the action principle of Fronsdal [39], is the content of the so called Central on-mass-shell theorem proved originally in $[35,3]$ for the $4 d$ case and then in $[36,37]$ for any dimension (see also [6] for more detail).

Note that the metric-like field of the Fronsdal formalism arises as the completely symmetric part of the frame field $\varphi_{\underline{m}_{1} \ldots \underline{m}_{s}}=e_{\left\{\underline{m}_{1}, \underline{m}_{2} \ldots \underline{m}_{s}\right\}}$, where all fiber indices have been lowered using the frame field $e_{0 \underline{m}}^{m}$. From the fiber index tracelessness of the on-shell HS frame field it follows automatically that the field $\varphi_{\underline{m}_{1} \ldots \underline{m}_{s}}$ is double traceless as required in the original Fronsdal formulation [39] (see [40] for pedagogical reviews of the Fronsdal formulation).

\section{$5 \quad s p(2)$ generators}

It is well known that the star product (3.2) describes the associative product of the Weyl ordered (i.e., totally symmetrized) polynomials of oscillators. The following formulae are simple consequences of the star product law (3.2)

$$
\begin{gathered}
{\left[p_{n}, f(p, y)\right]_{*}=\hbar \frac{\partial}{\partial y^{n}} f(p, y),} \\
\left\{p_{n}, f(p, y)\right\}_{*}=2 p_{n} f(p, y) .
\end{gathered}
$$

Using these relations along with the identity $\left[a^{2}, b\right]_{*} \equiv\left\{a,[a, b]_{*}\right\}_{*}$ one observes that

$$
\left[p^{2}, f\right]_{*}=2 \hbar p^{n} \frac{\partial}{\partial y^{n}} f=2 \hbar \tau_{+}(f) .
$$

More generally, the $s p(2)$ generators (4.29) admit the following realization in terms of the star product algebra

$$
\tau_{ \pm}(f)=\frac{1}{\hbar}\left[t_{ \pm}, f\right]_{*}, \quad \tau_{0}(f)=\frac{1}{\hbar}\left[t_{0}, f\right]_{*}
$$


with

$$
t_{-}=-\frac{1}{2} y_{n} y^{n}, \quad t_{+}=\frac{1}{2} p_{n} p^{n}, \quad t_{0}=y_{n} p^{n} .
$$

From the associativity of the star product it follows in particular that, given two lowest vectors $f$ and $g$ satisfying $\left[t_{-}, f\right]_{*}=\left[t_{-}, g\right]_{*}=0$, their star product is also a lowest vector: $\left[t_{-}, f * g\right]_{*}=\left[t_{-}, f\right]_{*} * g+f *\left[t_{-}, g\right]_{*}=0$.

In the subsequent analysis we will use the following simple lemmas:

\section{Lemma 1}

Any polynomial $X(p, y)$ can be uniquely decomposed as

$$
X(p, y)=\left.X(p, y)\right|_{V_{-}}+\tau_{+}\left(\left.X(p, y)\right|_{-}\right), \quad \tau_{-}\left(\left.X(p, y)\right|_{V_{-}}\right)=0 .
$$

Proof: Since the $s p(2)$ generators $\tau_{ \pm}$do not change a degree of a polynomial of $p$ and $y$, the space of functions $X(p, y)$ decomposes into an infinite direct sum of finite-dimensional $s p(2)$ submodules spanned by homogeneous polynomials of different degrees. Elements of these submodules can be generated by $\tau_{+}$from lowest weight vectors annihilated by $\tau_{-}$, i.e., any $X(p, y)$ can be represented as

$$
X(p, y)=\sum_{q \geq 0}\left(\tau_{+}\right)^{q} X_{q}(p, y), \quad \tau_{-} X_{q}(p, y)=0 .
$$

Then $\left.X(p, y)\right|_{V_{-}}=X_{0}(p, y)$ and $\left.X(p, y)\right|_{-}=\sum_{q \geq 1}\left(\tau_{+}\right)^{q-1} X_{q}(p, y)$.

Note that although $\tau_{+}\left(\left.X(p, y)\right|_{-}\right)$in (5.6) is defined uniquely, $\left.X(p, y)\right|_{-}$is defined modulo elements in $K \operatorname{er} \tau_{+}$. In what follows we will also use notation $\left.X\right|_{V_{+}}$and $\left.X\right|_{+}$for the decomposition analogous to (5.6) with the roles of $\tau_{-}$ and $\tau_{+}$exchanged.

Lemma 2

Let

$$
\tau_{+}(X(p, y))=0, \quad \tau_{0}(X(p, y))=\alpha X(p, y),
$$

where $\alpha$ is a number. Then

$$
\begin{aligned}
& \left.X(p, y)\right|_{V_{-}}=X, \quad \text { if } \quad \alpha=0, \\
& \left.X(p, y)\right|_{V_{-}}=0, \quad \text { if } \quad \alpha \neq 0 .
\end{aligned}
$$

This lemma follows from the fact that, for finite-dimensional $s p(2)$ modules, a lowest vector can the same time be a highest vector only for a trivial module. 
Lemma 3

Let

$$
\tau_{0} X^{+}(p, y)=Y^{+}(p, y),
$$

with $t^{-1} Y^{+}\left(t^{-1} p, t y\right)$ being a polynomial of $t$ or

$$
\tau_{0} X^{-}(p, y)=Y^{-}(p, y),
$$

with $t^{-1} Y^{-}\left(t p, t^{-1} y\right)$ being a polynomial of $t$. Then

$$
X^{+}(p, y)=\int_{0}^{1} d t t^{-1} Y^{+}\left(t^{-1} p, t y\right)
$$

and

$$
X^{-}(p, y)=-\int_{0}^{1} d t t^{-1} Y^{-}\left(t p, t^{-1} y\right)
$$

are particular solutions of the equations (5.11) and (5.12), respectively. We skip the proof as it is elementary.

\section{Linearization}

In this section we show that the nonlinear HS equations (3.4) reproduce correctly the off-shell HS system at the linearized level.

Let us analyze the equations (3.4) and the gauge transformations (3.12) perturbatively. Taking into account (3.7) and (3.8) and choosing Cartesian coordinates with $D^{L}=d$ and $e^{n}=d x^{n}$ one obtains to the first order

$$
\begin{gathered}
\left(d+d x^{n} \frac{\partial}{\partial y^{n}}\right) A_{1}(p, y \mid x)=0 \\
\tau_{+} A_{1}(p, y \mid x)=\left(d+d x^{n} \frac{\partial}{\partial y^{n}}\right) F_{1}(p, y \mid x),
\end{gathered}
$$

where we made use of (5.3).

The linearized gauge transformations are

$$
\begin{gathered}
\delta_{0} A_{1}(p, y \mid x)=\left(d+d x^{n} \frac{\partial}{\partial y^{n}}\right) \epsilon(p, y \mid x), \\
\delta_{0} F_{1}(p, y \mid x)=\tau_{+} \epsilon(p, y \mid x) .
\end{gathered}
$$

Let us now show that the equations (6.1) and (6.2) along with the gauge transformations (6.3) and (6.4) reproduce Central off-mass-shell theorem of subsection 4.3. To illustrate the idea we start with the lower spin examples of spin zero and spin one. 


\subsection{Spin zero and spin one}

For spin zero there is no gauge field $A$ and gauge symmetry parameter $\epsilon$. The spin zero part of the form $F$ is $p^{n}$-independent. The only nontrivial equation is therefore $(6.2)$ at $p^{n}=0$, i.e. $\left(d+d x^{n} \frac{\partial}{\partial y^{n}}\right) F_{1}(0, y \mid x)=0$, which is just the unfolded equation (2.38) of subsection 2.4.

In the spin one case we have $p$-independent gauge potential $d x^{\underline{n}} A_{\underline{n}}(y \mid x)$ and 0 -form $F_{1}$ linear in $p^{n}$, i.e

$$
F_{1}=\sum_{k=0}^{\infty} p^{n} y^{m_{1}} \ldots y^{m_{k}} F_{1 m_{1} \ldots m_{k} ; n} .
$$

The gauge transformation (6.4) with a $p$-independent gauge parameter has the form

$$
\delta F_{1 m_{1} \ldots m_{k} ; n}=\epsilon_{m_{1} \ldots m_{k} n}
$$

where $\epsilon_{m_{1} \ldots m_{l}}(x)$ is an arbitrary totally symmetric tensor. This means that the totally symmetric part in $F_{1 m_{1} \ldots m_{k} ; n}$ is pure gauge. Gauge fixing the totally symmetric part of $F_{1 m_{1} \ldots m_{k} ; n}$ to zero is equivalent to say that

$$
F_{1 m_{1} \ldots m_{k} ; n}=C_{m_{1} \ldots m_{l}, n}
$$

where the field $C_{m_{1} \ldots m_{l}, n}$ has the symmetry properties of the Young tableau

. These fields form the set of 0-forms which describe all off-shell gauge invariant derivatives of the spin one field as discussed in subsection 4.1. Thus, in this gauge

$$
F_{1}(p, y \mid x)=C(p, y \mid x), \quad \tau_{-}(C)=0,
$$

where $C(p, y \mid x)$ is the spin one 0-form (4.8) satisfying the condition (4.9).

The leftover gauge symmetry is described by the $y$-independent gauge parameter $\epsilon(x)$ which does not contribute to the variation of the spin one 0 -form $F_{1} . \epsilon(x)$ is the usual spin one gauge parameter.

Let us now apply the operator $\tau_{-}$to the both sides of (6.2), taking into account (6.8) and that $A(p, y \mid x)=A(y \mid x)$ is $p$-independent for spin 1. This gives

$$
y^{n} \frac{\partial}{\partial y^{n}} A_{1}(y \mid x)=-d x^{n} \frac{\partial}{\partial p^{n}} C(p, y \mid x),
$$

which means by Lemma 3 that

$$
A_{1}(y \mid x)=a(x)-d x^{n} \frac{\partial}{\partial p^{n}} \int_{0}^{1} d t t^{-1} C(p, t y \mid x),
$$


where $a(x)$ is an independent 1-form to be identified with the electromagnetic potential. Note that there is no pole in $t$ on the right hand side of the equation (6.10) because a field $C(p, y \mid x)$ satisfying (4.28) is at least linear in $y^{n}$ (since a Young tableau with one cell in the second row associated with $p^{m}$ must contain at least one cell in the first row associated with $\left.y^{n}\right)$. Plugging this back into the equation (6.2) one obtains

$$
\left(d+d x^{n} \frac{\partial}{\partial y^{n}}\right) C(p, y \mid x)+d x^{n} p^{m} \frac{\partial^{2}}{\partial p^{n} \partial y^{m}} \int_{0}^{1} d t t^{-1} C(p, t y \mid x)=0 .
$$

By an appropriate rescaling of the coefficients of the expansion of $C(p, y \mid x)$ in powers of $y$ and $p$ this reproduces the equation (4.5) for $s=1$.

Finally, it remains to plug (6.10) into (6.1). One observes that, applying the operator $\tau_{+}$to the both sides of (6.1) gives identity once the equation (6.2) is true. Therefore, a nontrivial part of (3.22) is contained in its $y^{n}$ independent sector. Setting $y=0$ in (6.1) one obtains

$$
d a(x)=\left.d x^{m} \wedge d x^{n} \frac{\partial^{2}}{\partial p^{n} \partial y^{m}} C(p, y)\right|_{p=y=0},
$$

which is equivalent to the equation (4.1). The equations (6.11) and (6.12) form the free off-shell spin one unfolded system.

\subsection{Any spin}

Let us now consider the general case. Taking into account that

$$
\delta_{0} F_{1}(p, y \mid x)=\tau_{+} \epsilon(p, y \mid x)
$$

and using Lemma 1 we see that it is possible to gauge away all components of $F_{1}(p, y)$ except for those of the form

$$
F_{1}(p, y \mid x)=C(p, y \mid x), \quad \tau_{-} C(p, y \mid x)=0
$$

i.e., $C(p, y \mid x)=\left.F_{1}(p, y \mid x)\right|_{V_{-}}$. The field $C(p, y \mid x)$ is just the generating function for the HS 0-forms satisfying the Young (anti)symmetry condition (4.28).

From (6.13) it also follows that the remaining gauge symmetry parameters satisfy the highest weight condition

$$
\tau_{+} \epsilon(p, y \mid x)=0
$$


i.e., the coefficients of the expansion

$$
\epsilon(p, y)=\sum_{p \geq q} \epsilon_{n_{1} \ldots n_{p}, m_{1} \ldots m_{q}} p^{n_{1}} \ldots p^{n_{p}} y^{m_{1}} \ldots y^{m_{q}}
$$

are described by two-row Young tableaux for which $p$ is associated with the first row and $y$ is associated with the second row. These are the gauge parameters of the frame-like formulation for symmetric HS fields [36] summarized in subsection 4.3 .

Let us now consider equation (6.2). It reconstructs $A_{1}(p, y \mid x)$ in terms of $C(p, y \mid x)$ modulo solutions of the homogeneous equation, that is

$$
A_{1}(p, y \mid x)=\omega(p, y \mid x)+\tilde{A}(p, y \mid x),
$$

where $\omega(p, y \mid x)$ is an arbitrary $s p(2)$ highest weight polynomial satisfying (4.27) to be identified with the HS gauge potentials, while $\tilde{A}$ is a particular solution of the equation (6.2). It is convenient to look for $\tilde{A}$ among the lowest weight vectors

$$
\tau_{-} \tilde{A}(p, y \mid x)=0 .
$$

Applying the operator $\tau_{-}$to the both sides of the equation (6.2) one obtains

$$
\tau_{0}(\tilde{A})=-d x^{n} \frac{\partial}{\partial p^{n}} C(p, y \mid x) .
$$

To solve this equation we apply Lemma 3 (5.11), (5.13), taking into account that, for $C(p, y \mid x)$ satisfying (6.14), a power of $p$ cannot be higher than that of $y$. This reconstructs $\tilde{A}$ in the form compatible with (6.18)

$$
A(p, y \mid x)=\omega(p, y \mid x)-d x^{n} \int_{0}^{1} d t \frac{\partial}{\partial p^{n}} C\left(t^{-1} p, t y \mid x\right) .
$$

Plugging this expression back into (6.2) one obtains

$$
\left(d+d x^{n} \frac{\partial}{\partial y^{n}}\right) C(p, y \mid x)+d x^{n} p^{m} \frac{\partial}{\partial y^{m}} \int_{0}^{1} d t \frac{\partial}{\partial p^{n}} C\left(t^{-1} p, t y \mid x\right)=0 .
$$

By an appropriate rescaling of the coefficients of the expansion of $C(p, y \mid x)$ in powers of $y$ and $p$ this reproduces the equation (4.24) for the chains of 0 -forms of any spin.

Finally, it remains to plug (6.20) into (6.1). Like in the spin one case, the application of the operator $\tau_{+}$to the both sides of (6.1) gives identity once (6.2) is true. This means that a nontrivial part of (6.1) is contained in the kernel of 
$\tau_{+}$. As a result, it is easy to see that the nontrivial part of the equation (6.1) is

$$
d \omega(p, y \mid x)+d x^{n} \frac{\partial}{\partial y^{n}} \omega(p, y \mid x)=d x^{m} \wedge d x^{n} \frac{\partial^{2}}{\partial p^{n} \partial y^{m}} C^{l}(p, y \mid x),
$$

where $C^{l}(p, y \mid x)$ is the lowest tensor part of $C(p, y \mid x)$ that is annihilated both by $\tau_{-}$and by $\tau_{+}$thus being a $\operatorname{sp}(2)$ singlet

$$
\tau_{-} C^{l}(p, y \mid x)=0, \quad \tau_{+} C^{l}(p, y \mid x)=0, \quad \tau_{0} C^{l}(p, y \mid x)=0,
$$

which means that it is described by a rectangular Young tableau, i.e. $C^{l}(p, y \mid x)$ is the Maxwell tensor for $s=1$, Riemann tensor for $s=2$ and their HS analogues for higher spins. (Note that $C^{l}\left(t^{-1} p, t y \mid x\right)=C^{l}(p, y \mid x)$.) The equation (6.22) reproduces the chain of constraints (4.23). Thus, the equations (6.22), (6.21) form the unfolded off-shell system for a free spin $s$ massless field provided that $\omega(p, y \mid x)$ and $C(p, y \mid x)$ are, respectively, of degrees $s-1$ and $s$ in $p^{n}$.

Let us note that the analysis of this section is algebraically analogous to the analysis of free HS fields performed in [16] within the BRST approach, where $s p(2)$ generators (constraints) also play the key role. In particular, the fact that the on-shell version of the equations (6.1) and (6.2) describes properly linearized HS dynamics was shown by the authors of [16]. The important difference is that the analysis of [16] uses the standard BRST language of "states" where the BRST operator acts, that requires a doubled number of oscillators compared to our approach where "states" are replaced by the associative star product algebra. Among other things, the advantages of our formalism are that all formulae remain valid in any coordinate system in Minkowski space by replacing de Rham differential $d$ with the Lorentz covariant derivative $D^{L}$ and $d x^{n}$ by the frame 1 -form $e^{n}$ and, most important, that it admits a natural generalization to the interacting case.

\section{$7 \quad$ Nonlinear off-shell analysis}

To reproduce unfolded HS constraints it is important in our approach that $A(p, y \mid x)$ and $F(p, y \mid x)$ contain the zero-order parts (3.7) and (3.8). In the nonlinear analysis we will not single out the vacuum part of the gravitational field, setting

$$
A(p, y \mid x)=d x^{\underline{n}}\left(e_{\underline{n}}^{n}(x) p_{n}+\omega_{\underline{n}}^{n m}(x) p_{n} y_{m}+A_{\underline{n} 1}(p, y \mid x)\right)
$$




$$
F(p, y \mid x)=t_{+}+F_{1}(p, y \mid x) .
$$

Here $e_{\underline{n}}^{n}(x) p_{n}, \omega_{\underline{n}}^{n m}(x) p_{n} y_{m}$ and of course the flat Minkowski metric $\eta^{m n}$ in $t_{+}$ are not supposed to be small ${ }^{7}$. The fields $A_{\underline{n} 1}(p, y \mid x)$ and $F_{1}(p, y \mid x)$ are treated as fluctuations. $A_{\underline{n} 1}(p, y \mid x)$ does not include the gravitational part associated with the fields $e^{n}$ and $\omega^{m n}$ in (7.1), i.e we require

$$
\left.A_{\underline{n} 1}(p, y \mid x)\right|_{V_{+}}=0 \quad \text { if } \quad\left(p^{n} \frac{\partial}{\partial p^{n}}-1\right) A_{\underline{n} 1}(p, y \mid x)=0 .
$$

The gauge transformation law is

$$
\begin{gathered}
\delta A(p, y \mid x)=D^{L} \epsilon(p, y \mid x)+e^{n} \frac{\partial}{\partial y^{n}} \epsilon(p, y \mid x)+\left[A_{1}(p, y \mid x), \epsilon(p, y \mid x)\right]_{*}, \\
\delta F_{1}(p, y \mid x)=\tau_{+}(\epsilon(p, y \mid x))+\left[F_{1}(p, y \mid x), \epsilon(p, y \mid x)\right]_{*},
\end{gathered}
$$

where $D^{L}$ is the Lorentz covariant derivative

$$
D^{L}=d+\omega^{m n}\left(y_{n} \frac{\partial}{\partial y^{m}}+p_{n} \frac{\partial}{\partial p^{m}}\right) .
$$

Since the gauge transformation law (7.5) has the form (6.13) in the leading order, it is still possible to impose the gauge condition (6.14), which we write in the form

$$
F_{1}(p, y \mid x)=C(p, y \mid x), \quad\left[t_{-}, C\right]_{*}=0 .
$$

The field equations (3.4) now read as

$$
\begin{aligned}
& R^{m n} p_{m} y_{n}+R^{m} p_{m}+D^{L} A_{1}(p, y \mid x)+e^{n} \frac{\partial}{\partial y^{n}} A_{1}(p, y \mid x)+\left(A_{1} * \wedge A_{1}\right)(p, y \mid x)=0, \\
& D^{L} C(p, y \mid x)+e^{n} \frac{\partial}{\partial y^{n}} C(p, y \mid x)-\tau_{+}\left(A_{1}(p, y \mid x)\right)+\left[A_{1}(p, y \mid x), C(p, y \mid x)\right]_{*}=0,
\end{aligned}
$$

where $R^{m}$ and $R^{m n}$ are, respectively, the torsion tensor (2.13) and the Riemann tensor (2.14).

Since the equation (7.8) is the compatibility condition for (7.9), a significant part of the information contained in (7.8) is a consequence of that contained

\footnotetext{
${ }^{7}$ Of course, this requirement is needed when discussing a phase with a nondegenerate metric tensor as in the space we used to live in. One can in principle think of different phases where what we call gravitational metric field is degenerate. This interesting option is in fact very much in spirit of the unfolded dynamics and may be related to models with invisible extra dimensions as discussed in [20].
} 
in (7.9). Let us show that a part of the equation (7.8) independent of (7.9) belongs to $\operatorname{Ker} \tau_{+}$. The proof is by induction.

Actually, suppose that the content of the equation (7.8) has been checked up to an order $q$ in powers of $F_{1}=C$, while that of the equation (7.9) has been checked up to the order $q+1$. (One can choose $q=-1$ as the starting point in which case the equation (7.9) implies that $A_{1}(p, y \mid x)$ belongs to $\operatorname{Ker} \tau_{+}$.) Consider the order $q+1$ part of the equation

$$
[F,(d+A) \wedge(d+A)]_{*}=0 \text {. }
$$

Since this is a consequence of the equation

$$
d F+[A, F]_{*}=0
$$

which by assumption is true up to an order $q+1$ in $C$, it follows that the equation (7.10) is also satisfied up to the same order. Taking into account (7.1), where $F_{1}(p, y \mid x)$ is of order one, and that the equation $(d+A) \wedge(d+A)=0$ was by assumption analysed up to order $q$ it follows from (7.10) that

$$
\tau_{+}((d+A) \wedge *(d+A))=0
$$

is true up to order $q+1$ as a consequence of the other equations.

Thus, the only nontrivial part of the equation (7.8) that remains to be analysed is that in $\operatorname{Ker} \tau_{+}$. It can be written in the form

$R^{m n} p_{m} y_{n}+R^{m} p_{m}+\left.\left(D^{L} A_{1}(p, y \mid x)+e^{n} \frac{\partial}{\partial y^{n}} A_{1}(p, y \mid x)+\left(A_{1} * \wedge A_{1}\right)(p, y \mid x)\right)\right|_{V_{+}}=0$

So, the equations (7.9) and (7.13) are equivalent to the equations (3.4) with the gauge condition (7.7). Let us note that the general analysis remains true if all fields take values in a matrix algebra (i.e., carry matrix indices) provided that the gravitational field lies in its center (i.e., is proportional to the unit element of this matrix algebra).

Before considering the general case, let us discuss the important lower spin examples of gravity, Yang-Mills theory and a scalar field.

\section{$7.1 s \leq 2$}

What simplifies the analysis of the lower spins $s \leq 2$ is that the property (6.18) of the linearized analysis remains true in all orders. Indeed, taking into account (7.7), the algebraic part of the equation (7.9), that does not contain 
$D C(p, y \mid x)$, results from the application of $\tau_{-}$to the both sides of (7.9). This gives

$$
\begin{aligned}
\tau_{0}\left(A_{1}(p, y \mid x)\right) & =-e^{n} \frac{\partial}{\partial p^{n}} C(p, y \mid x)-\tau_{+}\left(\tau_{-}\left(A_{1}(p, y \mid x)\right)\right) \\
& +\left[\tau_{-}\left(A_{1}(p, y \mid x)\right), C(p, y \mid x)\right]_{*}=0 .
\end{aligned}
$$

One can now iterate this equation to reconstruct $A_{1}(p, y \mid x)$ in terms of $C(p, y \mid x)$. According to the original equation (7.9), it should reconstruct $A_{1}(p, y \mid x)$ in terms of $C(p, y \mid x)$ up to zero modes of $\tau_{+}$identified with the dynamical gauge fields $\omega(p, y \mid x)$. The appearance of the latter fields in the last term on the right hand side of (7.14) does not allow us to find a solution with $A_{1}(p, y \mid x)$ being a lowest weight element for the general spin. For spin two, however, the zero mode has been already separated into $e^{n}$ and $\omega^{n m}$ so that, by the condition (7.3), it is not contained in $A_{1}(p, y \mid x)$. For spin one, the zero mode $a(x)$ is independent of $p^{n}$ and $y^{n}$, i.e. it itself satisfies the lowest weight condition in a trivial way.

As a result, for spins $s \leq 2$ we solve (7.14) the same way as in the linear problem to obtain

$$
A_{1}(p, y \mid x)=a(x)-e^{n} \int_{0}^{1} d t \frac{\partial}{\partial p^{n}} C\left(t^{-1} p, t y \mid x\right),
$$

where $a(x)$ is the Yang-Mills gauge field. The last two terms in (7.14) drop out because

$$
\tau_{-}\left(A_{1}(p, y \mid x)\right)=0 .
$$

Let us stress that the analysis of this section works for the non-Abelian case where $a(x)$ and all spin one 0 -forms (i.e., those linear in $p^{n}$ ) take values in a Yang-Mills algebra $g$, while the spin zero 0 -forms independent of $p$ are in some representation of $g$.

To analyse the content of the equation (7.13) we observe that a $s p(2)$ singlet part $a(x)$ of the solution (7.15) is the Yang-Mills field. The $C$-dependent part in (7.15) belongs to $\operatorname{Ker} \tau_{-}$but contains no singlets because its degree in $y$ is strictly higher than that of $p$ as a result of differentiation over $p$ in (7.15), that means that it contains only eigenvectors of $\tau_{0}$ with strictly positive eigenvalues. Taking into account Lemma 2 along with the properties that $(i)$ star product of two elements $a_{1,2}$ satisfying the lowest weight conditions $\left[t_{-}, a_{1,2}\right]_{*}=0$, is also lowest weight and $(i i)\left[t_{0}, a_{1} * a_{2}\right]_{*}=\left(\alpha_{1}+\alpha_{2}\right) a_{1} * a_{2}$ if $\left[t_{0}, a_{i}\right]_{*}=\alpha_{i} a_{i}$ $(\mathrm{i}=1,2)$, we observe that all $C$-dependent terms in $A_{1}$ do not contribute to (7.13) except for the term $e^{n} \frac{\partial}{\partial y^{n}} A_{1}(p, y \mid x)$ that decreases the $y$ degree by one 
unit. The terms with the Yang-Mills connection $a(x)$ form the Yang-Mills field strength

$$
R(x)=d a(x)+a(x) \wedge a(x) .
$$

As a result, the equation (7.13) simplifies to

$$
R^{m n}(x) p_{m} y_{n}+R^{m}(x) p_{m}+R(x)=e^{n} \wedge e^{m} \frac{\partial^{2}}{\partial y^{n} \partial p^{m}} C^{l}(p, y \mid x),
$$

where $C^{l}(p, y \mid x)$ is the $s p(2)$ singlet part of the 0 -form $C(p, y \mid x)$ described by rectangular Young tableaux, so that for the spins two, one and zero

$$
C^{l}(p, y \mid x)=C^{k l, n m}(x) y_{k} y_{l} p_{n} p_{m}+C^{n, m}(x) y_{n} p_{m}+C(x) .
$$

The spin two and spin one parts contribute to the equation (7.18). The meaning of this equation is now obvious. It implies that torsion tensor is zero, thereby expressing the Lorentz connection via the frame field $e^{n}$, and identifies $C^{n m, k l}(x)$ and $C^{n, m}(x)$ with the components of the Riemann and Yang-Mills curvatures imposing no conditions on the latter.

Now we plug the expression (7.15) into the equation (7.9). The terms with the Yang-Mills connection form the full Lorentz-Yang-Mills derivative

$$
D^{L Y M}=D^{L}+[a, \ldots] .
$$

The equation (7.9) now takes the form

$$
\begin{aligned}
D^{L Y M} C(p, y \mid x) & +e^{n} \int_{0}^{1} d t\left(\frac{\partial}{\partial y^{n}} C(p, y \mid x)+\tau_{+}\left(\frac{\partial}{\partial p^{n}} C\left(t^{-1} p, t y \mid x\right)\right)\right. \\
& \left.\left.-\left[\frac{\partial}{\partial p^{n}} C\left(t^{-1} p, t y \mid x\right)\right), C(p, y \mid x)\right]_{*}\right)=0 .
\end{aligned}
$$

This equation provides a fully consistent nonlinear deformation of the linearized equation (4.24). Together with (7.18) it gives the unfolded form of nonlinear off-shell constraints for the fields of spins two, one and zero.

Let us mention a subtle point. In the formulation with the star product we use, there is a contribution of the terms built of the spin two Riemann tensor and its derivatives to the sector of spin zero 0-forms in the equation (7.21), which is

$$
\left.\hbar^{2} e^{n} \frac{\partial^{4}}{\partial p^{n} \partial p^{m} \partial y^{k} \partial y^{l}} C(p, y \mid x) \frac{\partial^{3}}{\partial y_{m} \partial p_{k} \partial p_{l}} C(p, y \mid x)\right|_{p=0} .
$$

Although this term disappears in the "semiclassical" limit $\hbar \rightarrow 0$ and can therefore be consistently neglected off-shell, its appearance indicates that HS fields form sources for lower spin fields which effect looks to be inevitable in the on-shell theory in $A d S_{d}$ where it is not possible to take a semiclassical limit of the star product algebra. 


\subsection{Any spin}

Using the decomposition (5.6) we obtain from (7.9) two equations. One is the differential equation on $C(p, y \mid x)$

$$
D^{L} C(p, y \mid x)+\left.e^{n}\left(\frac{\partial}{\partial y^{n}} C(p, y \mid x)\right)\right|_{V_{-}}+\left.\left(\left[A_{1}(p, y \mid x), C(p, y \mid x)\right]_{*}\right)\right|_{V_{-}}=0 .
$$

Another one

$$
\tau_{+}\left(A_{1}(p, y \mid x)\right)=\tau_{+}\left(\left.\left(e^{n} \frac{\partial}{\partial y^{n}} C(p, y \mid x)+\left[A_{1}(p, y \mid x), C(p, y \mid x)\right]_{*}\right)\right|_{-}\right)
$$

is the algebraic equation that reconstructs the gauge connection $A_{1}(p, y \mid x)$ in terms of $C(p, y \mid x)$ and HS gauge connections $\omega(p, y \mid x)$ satisfying (4.27).

Since $\tau_{-}(C)=0$, the following identity is true

$$
\tau_{-}\left(e^{n} \frac{\partial}{\partial y^{n}} C(p, y \mid x)+\tau_{+}\left(\left(\tau_{0}\right)^{-1} e^{n} \frac{\partial}{\partial p^{n}} C(p, y \mid x)\right)\right)=0
$$

which means that

$$
\begin{gathered}
\left.\left.\left(e^{n} \frac{\partial}{\partial y^{n}} C(p, y \mid x)\right)\right|_{V_{-}}=e^{n} \frac{\partial}{\partial y^{n}} C(p, y \mid x)\right)+\tau_{+}\left(\left(\tau_{0}\right)^{-1} e^{n} \frac{\partial}{\partial p^{n}} C(p, y \mid x)\right) \\
\left.\tau_{+}\left(e^{n} \frac{\partial}{\partial y^{n}} C(p, y \mid x)\right)\right|_{-}=-\tau_{+}\left(\left(\tau_{0}\right)^{-1} e^{n} \frac{\partial}{\partial p^{n}} C(p, y \mid x)\right)
\end{gathered}
$$

By virtue of these identities and using Lemma 3 one obtains

$$
\begin{gathered}
\left.D^{L} C(p, y \mid x)+e^{n} \frac{\partial}{\partial y^{n}} C(p, y \mid x)\right)+\tau_{+}\left(e^{n} \int_{0}^{1} d t\left[\frac{\partial}{\partial p^{n}} C\left(t^{-1} p, t y \mid x\right)\right)\right. \\
+\left.\left(\left[A_{1}(p, y \mid x), C(p, y \mid x)\right]_{*}\right)\right|_{V_{-}}=0 \\
A_{1}(p, y \mid x)=\omega(p, y \mid x)-e^{n} \int_{0}^{1} d t \frac{\partial}{\partial p^{n}} C\left(t^{-1} p, t y \mid x\right)+\left.\left(\left[A_{1}(p, y \mid x), C(p, y \mid x)\right]_{*}\right)\right|_{-}
\end{gathered}
$$

where $\omega(p, y \mid x)$ is the generating function for HS gauge fields, i.e. an arbitrary 1 -form such that $\tau_{+}(\omega(p, y \mid x))=0$. The equation (7.29) determines $A_{1}(p, y \mid x)$ perturbatively as an expansion in powers of the HS gauge field $\omega(p, y \mid x)$ and the Weyl 0-forms $C(p, y \mid x)$. The equation (7.28) is the unfolded equation on $C(p, y \mid x)$. 
Inserting (7.29) into (7.13) one obtains a nonlinear differential equation on $\omega(p, y \mid x)$ of the form

$$
\begin{aligned}
R^{m n} p_{m} y_{n}+R^{m} p_{m} & +D^{L} \omega(p, y \mid x)+\omega(p, y \mid x) * \wedge \omega(p, y \mid x) \\
& =e^{n} \wedge e^{m} \frac{\partial^{2}}{\partial p^{m} \partial y^{n}} C^{l}(p, y \mid x)+O(\omega, C),
\end{aligned}
$$

where $O(\omega, C)$ contains at least one power of $C$ and is at least bilinear in $\omega$ and/or $C$. These terms are nonlinear because both $C$ and $\omega$ (which does not contain the gravitational gauge fields) are treated as small perturbations.

The equations (7.30) and (7.28) provide nonlinear unfolded off-shell constraints for all HS fields in flat space. All nonlinear corrections can be reconstructed order by order by iterations of the equation (7.29). The consistency is guaranteed by construction.

\section{Nonlinear unfolded equations for lower spins}

Now let us discuss a possibility of putting off-shell systems on-shell by imposing nontrivial field equations. At the linearized level it is well-known [36, 37] (see also [6]) that to put a system on-shell it is enough to require the gauge fields and field strengths to be traceless in the fiber indices. It is also well-known [41] that it is not possible to extend the on-shell formulation for massless spins $s>2$ to the nonlinear level unless a nonzero cosmological constant is introduced [7], for which case the problem was solved in [1, 2] (and references therein). On the other hand, since the full off-shell system admits consistent lower spin reductions and the latter admit consistent nonlinear field equations like YangMills and Einstein equations, it is interesting to see how these equations look like in terms of the unfolded formulation suggested in this paper. As we show in this section the lower spin equations keep the standard form in the unfolded formulation although formulated in terms of 0 -forms $F$ in place the original 1-form gauge fields $A$.

\subsection{Yang-Mills theory}

Yang-Mills theory is described in terms of the gauge 1-form $A$ and Weyl zero form $F$ having the form (3.6) with the background gravitational fields $A_{0}(3.7)$ and $F_{0}(3.8)$ and dynamical spin 1 fields $A_{1}=A_{1}(y \mid x)$ and $F_{1}=p_{n} F_{1}^{n}(y)$ of zero and first order in $p_{n}$, respectively. Here the fields $A_{1}=A_{1}(y \mid x)$ and $F_{1}=$ 
$p_{n} F_{1}^{n}(y)$ take values in a Yang-Mills Lie algebra as well as the $p$-independent gauge parameter $\epsilon(y \mid x)$.

The key observation is that, because of the vacuum part $F_{0}(3.8)$, the spin one 0 -form $F_{n}$ transforms as Yang-Mills connection in the $y$-space

$$
\delta F_{n}(y \mid x)=D_{n}^{y} \epsilon(y \mid x)
$$

where

$$
D_{m}^{y} \epsilon(y \mid x)=\frac{\partial}{\partial y^{m}} \epsilon(y \mid x)+\left[F_{m}(y \mid x), \epsilon(y \mid x)\right]
$$

As a result,

$$
F^{m n}(y \mid x)=\frac{\partial}{\partial y_{m}} F^{n}(y \mid x)-\frac{\partial}{\partial y_{n}} F^{m}(y \mid x)+\left[F^{m}(y \mid x), F^{n}(y \mid x)\right]
$$

behaves as $y$-space field strength and transforms covariantly

$$
\delta F_{n m}(y \mid x)=\left[F_{n m}(y \mid x), \epsilon(y \mid x)\right] .
$$

The $x$-space Yang-Mills equations result from those in the $y$ space

$$
D_{n}^{y} F^{n m}(y \mid x)=0 \text {. }
$$

Although this conclusion may look like a miracle, it is just a consequence of the identical form of the Yang-Mills symmetries in the $x$-space and $y$-space.

Analogously to the case of scalar field it is not difficult to write a general $Q$ closed Lagrangian for spin one

$$
\mathcal{L}=\left.\epsilon_{n_{1} \ldots n_{d}} e^{n_{1}} \wedge \ldots \wedge e^{n_{d}} \ell\left(F_{n m}, D_{k}^{y} F_{n m}, \ldots\right)\right|_{y=0}
$$

where $\ell\left(F_{n m}, D_{k}^{y} F_{n m}, \ldots\right)$ is a Lagrangian function of the $y$-field strength $F^{m n}$ and its $y$-covariant derivatives taken at $y=0$, which is invariant under the $y$-dependent Yang-Mills symmetry transformations. In particular, the Lagrangian of the standard Yang-Mills theory has the form

$$
\mathcal{L}=\epsilon_{n_{1} \ldots n_{d}} e^{n_{1}} \wedge \ldots \wedge e^{n_{d}} \operatorname{tr}\left(F^{n m}(0 \mid x) F_{n m}(0 \mid x)\right),
$$

where $t r$ is trace in a chosen matrix representation of the Yang-Mills algebra. This Lagrangian is manifestly gauge invariant under the gauge transformations with the parameters $\epsilon(y \mid x)$ and therefore is $Q$ closed. That it is equivalent to the standard Yang-Mills Lagrangian follows from the fact that, taking into account that the nonlinear term in (8.3) is at least linear in $y$,

$$
F^{n m}(0 \mid x)=\left.\left(\frac{\partial}{\partial y_{m}} F^{n}(y \mid x)-\frac{\partial}{\partial y_{n}} F^{m}(y \mid x)\right)\right|_{y=0}=2 C^{n, m}(x),
$$

where $C^{n, m}(x)$ is Maxwell 0 -form equal to the $x$-space Yang-Mills field strength by virtue of the $p$-independent part of (7.18). 


\subsection{Gravity}

To describe gravity one observes that the 0 -form $F^{n m}(y \mid x)$ transforms like the metric tensor under the $y$-diffeomorphisms (3.19). Since it starts with the flat part

$$
F^{n m}(y \mid x)=\frac{1}{2} \eta^{n m}+F_{1}^{n m}(y \mid x)
$$

the matrix $F^{n m}(y \mid x)$ is (perturbatively) invertible. The invariant conditions on $F^{n m}(y \mid x)$ have a form of standard Einstein equations in the $y$ space

$$
\mathcal{R}_{n m}(F(y \mid x))=0, \quad \mathcal{R}_{n m}=\mathcal{R}_{n k, m l} F^{k l},
$$

where $\mathcal{R}_{n m, k l}(F)$ is the Riemann tensor constructed from $F^{n m}(y \mid x)$ treated as the metric tensor in the $y$-space. Containing second derivatives, the $y-$ space Einstein equations (8.10) impose conditions on the components of the $y$-space Riemann tensor $F_{p q}^{n m} y^{p} y^{q}$ and higher order polynomials in $y$. The zero curvature equations of the unfolding approach map these conditions to the true Einstein equations on the frame $e^{n}$ where $F_{p q}^{n m}$ is identified with the $x$-space Riemann tensor by (7.18).

Note that the expansion in powers of $y^{n}$ is a version of the normal coordinate expansion. Also let us note that such a mechanism does not work for second-order HS equations of massless fields because the nontrivial components of the spin $s$-forms start from order- $s$ derivatives of the HS potentials $\omega(p, y \mid x)$. This is in agreement with the expectation that HS massless fields do not admit nontrivial interactions in flat Minkowski background [41]. On the other hand, one can speculate that the problem can be avoided even in flat space within the nonlocal approach developed in [42].

A general Lagrangian for the case of gravity can be written in the form

$$
\begin{aligned}
\mathcal{L}= & \epsilon_{n_{1} \ldots n_{d}} e^{n_{1}} \wedge \ldots \wedge e^{n_{d}} \sqrt{(-1)^{d+1} \epsilon^{m_{1} \ldots m_{d}} \epsilon^{k_{1} \ldots k_{d}} F_{k_{1} m_{1}} F_{k_{2} m_{2}} \ldots F_{k_{d} m_{d}}} \\
& \left.\ell\left(F^{n m}, \mathcal{R}_{n m, k l}, D_{p}^{y} \mathcal{R}_{n m, k l}, \ldots\right)\right|_{y=0} .
\end{aligned}
$$

This Lagrangian is invariant under the $y$-diffeomorphisms and (therefore) is $Q$ closed provided that all indices in $\ell\left(F^{n m}, \mathcal{R}_{n m, k l}, D_{p} \mathcal{R}_{n m, k l}, \ldots\right)$ are contracted covariantly. Indeed, because all quantities in the Lagrangian (8.11) transform covariantly under $y$-diffeomorphisms and because the Lagrangian is taken at $y=0$, only the global $G L(d)$ part of $y$-space diffeomorphisms acts nontrivially in (8.11). This $G L(d)$ part rotates the fiber indices, leaving the Lagrangian invariant provided that all indices are contracted covariantly. The only subtlety is that, although the epsilon symbol $\epsilon^{m_{1} \ldots m_{d}}$ is only $S L(d)$ covariant, the 
combination of the epsilon symbols with upper and lower indices in (8.11) is $G L(d)$ covariant.

The Einstein action results from

$$
\ell=F^{n m} \mathcal{R}_{n m}
$$

as is elementary to see in the gauge (6.14) in which the spin two fluctuational part $F_{1}^{n m}(y \mid x)$ carries at least two powers of $y$. In this gauge, the $y$-independent part of $F^{n m}(y \mid x)$ is just the constant term in (8.9). As a result, the square root in (8.11) becomes a constant. Analogously to the Yang-Mills case, the $y$-space Riemann tensor at $y=0$ is given by the expression linear in the Riemann 0 -form $F_{p q}^{n m}$ identified with the $x$-space Riemann tensor by (7.18).

\section{Contractions}

The property that HS fields form infinite towers follows from the structure of the star product algebra. However, because no obstruction for unfolding Bianchi identities with no dynamical equations imposed can be expected, it should be possible to unfold any off-shell HS system in the background gravitational and/or Yang-Mills fields. In practice, this is achieved by replacing the star product algebra with the commutative algebra of functions of commuting variables $y^{n}$ and $p_{n}$ in the spin $s \neq 2$ sector, keeping the Poisson algebra in the spin two sector of connections linear in $p$. In other words, one considers the semidirect product of the commutative associative algebra $H$ spanned by the polynomials $P(p, y)$ of all powers in $p^{n}$ except for linear polynomials (clearly polynomials linear in $p$ never appear in a product of any two such functions). Then one considers a Lie algebra $t$ of linear in $p$ polynomials formed by their Weyl commutators or, that is equivalent for the linear functions in $p$ (i.e., vector fields), by the Poisson brackets

$$
\left\{p_{m}, y^{n}\right\}=\delta_{m}^{n}
$$

resulting from the "classical limit" of (3.1). This gives rise to the spin two gauge fields. Then one defines the action of $t$ on $H$ as a Poisson bracket

$$
a(f)=\{a, f\} \quad \forall a \in t, \quad f \in H .
$$

Note that from this definition it follows that $a(f)$ has the same power in $p_{m}$ as $f(p, y)$, i.e. in this algebraic setup different spins do not mix. The unfolded 
equations keep the same form (3.4) but with the commutator replaced with the Poisson bracket with the spin two connection $A(p, y \mid x)$ linear in $p$. In addition, one can introduce the non-Abelian Yang-Mills structure by allowing the spin one (i.e., $p$-independent) 1-form gauge potentials to be matrices that allows for a Yang-Mills covariantization by extending the algebra to the semidirect sum of the HS commutative algebra with the spin two - one non-Abelian algebra. In this construction, off-shell HS fields are independent and may carry any representations of the Yang-Mills algebra. Moreover, in this setup it is possible to consider any number of HS fields of any spin. For example: an off-shell system of gravity, Yang-Mills theory with the Yang-Mills algebra $h$ and a spin three field in any given representation of $h$ is possible. (That, of course, in no way means that this system can be put on shell.)

There is also an intermediate truncation of the system (3.4) with the star product replaced with the Poisson brackets (9.1). All these off-shell formulations are formally consistent. Moreover, we admit that they even should be equivalent by an appropriate pseudolocal field redefinition analogous to the Seiberg-Witten map [43] (more precisely, an off-shell system is expected to be equivalent to its further contraction with the same spectrum of fields).

To impose dynamical equations in these terms means to restrict somehow the 1-form connection $A(p, y \mid x)$ and the 0 -form $F(p, y \mid x)$. Because the system (3.4) is overdetermined it is not trivial to do it in a consistent way. Here is where the role of $A d S$ background and star product structure becomes really important. The original star product version of the system seems to be fundamental because it is inherited from the fundamental system in $A d S_{d}$ of [2] that admits nontrivial field equations.

\section{Conclusion}

In this paper the general unfolded dynamics approach is extended to the action level for off-shell unfolded systems. Analogous construction for the on-shell unfolded systems gives conserved charges. Both types of objects are represented by cohomology of the derivation $Q$ of the $L_{\infty}$ algebra associated with the unfolded system at hand.

Also we present a very simple unfolded form for off-shell symmetric bosonic HS fields of all spins in Minkowski space. The constraints have a form of covariant constancy and zero curvature conditions for 1-forms and 0-forms taking values in an appropriate star product algebra. The form of these equations is very suggestive. Their extension to fermionic systems seems to be straightfor- 
ward along the lines of [44] and will be given elsewhere.

The off-shell HS system in Minkowski space, considered in this paper, results from the flat limit of the AdS HS system of [2]. Details of this correspondence will be given in a future publication. Let us just mention that the 0 -form $F$ of this paper is one of the covariantly constant $s p(2)$ generators that underly the construction of [2].

An interesting problem for the future is to apply the general scheme proposed in this paper to the construction of the action principle in the AdS HS gauge theory. Also it is interesting to extend the obtained results to superstring. It is tempting to speculate that the generators of $s p(2)$, which play the key role both in the $A d S$ analysis of [2] and in the Minkowski analysis of this paper, should extend to the Virasoro algebra via extension of a pair of oscillators $p^{n}, y_{n}$ to the infinite set $p_{i}^{n}, y_{i}^{n}$ with $i=0,1,2 \ldots$ a la the Moyal formulation of superstring [45].

\section{Acknowledgments}

I am grateful to M.Grigoriev, E.Ivanov and I.Tipunin for useful discussions. The work was supported in part by grants RFBR No. 05-02-17654, LSS No. 1578.2003-2 and INTAS No. 03-51-6346.

\section{Appendix A.}

\section{Unfolded equations with manifest $x$-dependence}

The unfolded equations also make sense when the differential $Q$ and/or dynamical variables on which it acts contain the manifest dependence on the coordinates $x^{\underline{n}}$ on the top of that due to $W^{\alpha}(x)$. In this case we set

$$
Q=\partial+G^{\alpha}(W, x) \frac{\partial}{\partial W^{\alpha}},
$$

where the partial exterior differential $\partial=d x^{\underline{n}} \frac{\partial}{\partial x^{\underline{n}}}$ takes into account only on the manifest dependence on $x$, i.e.

$$
\partial(F(W(x), x))=\left.\left(d x^{\underline{n}} \frac{\partial}{\partial x^{\underline{n}}} F(W, x)\right)\right|_{W=W(x)} .
$$

The condition $Q^{2}=0$ takes the form

$$
\partial G^{\alpha}(W, x)+G^{\beta}(W, x) \wedge \frac{\partial G^{\alpha}(W, x)}{\partial W^{\beta}}=0 .
$$


The equation (2.7) extends to

$$
d F(W(x), x)=Q(F(W(x), x))
$$

One can see that it remains invariant under the gauge transformations (2.8). Note also that for $W$-independent functions $F=F(x)$ the equation (A.4) reduces to the identity $d F(x)=\partial F(x)$.

\section{Appendix B.}

\section{Dynamical content via $\sigma_{-}$cohomology}

In this Appendix, we perform following $[18,21,9,6]$ a very general analysis of equations of motion of the form

$$
\widehat{D}_{0} \mathcal{C}=0
$$

via a cohomological reformulation of the problem. Here $\mathcal{C}$ is a differential form of degree $p$ taking values in a vector space $V$, that is an element of the complex $V \otimes \Omega^{p}\left(\mathcal{M}^{d}\right)$. We require $V$ to admit a grading operator $G$ and $\widehat{D}_{0}$ to decompose as the sum

$$
\widehat{D}_{0}=\sigma_{-}+D_{0}+\sigma_{+}
$$

such that the following properties are true:

(i) The grading operator $G$ is diagonalizable in the vector space $V$ and it possesses a spectrum bounded from below.

(ii)

$$
\left[G, D_{0}^{L}\right]=0, \quad\left[G, \sigma_{-}\right]=-\sigma_{-} .
$$

The operator $\sigma_{+}$is a sum of operators of strictly positive grade.

(iii) The operator $\sigma_{-}$acts vertically in the fibre $V$, i.e. it does not act on space-time coordinates. (In HS models, only the operator $D_{0}$ acts nontrivially on the space-time coordinates (differentiates).)

(iv) The operator $\widehat{D}_{0}$ (B.2) is nilpotent

$$
\left(\widehat{D}_{0}\right)^{2}=0 .
$$

and increases a form degree by one. 
The graded decomposition of the nilpotency equation gives the following identities

$$
\left(\sigma_{-}\right)^{2}=0, \quad D_{0} \sigma_{-}+\sigma_{-} D_{0}=0, \quad\left(D_{0}\right)^{2}+\sigma_{+} \sigma_{-}+\sigma_{-} \sigma_{+}+D_{0} \sigma_{+}+\sigma_{+} D_{0}+\left(\sigma_{+}\right)^{2}=0 .
$$

If $\sigma_{+}$has definite grade +1 , the last relation is equivalent to the three conditions $\left(\sigma_{+}\right)^{2}=0, \quad D_{0} \sigma_{+}+\sigma_{+} D_{0}=0, \quad\left(D_{0}\right)^{2}+\sigma_{+} \sigma_{-}+\sigma_{-} \sigma_{+}=0$. An important property is the nilpotency of $\sigma_{-}$. The key fact is that the analysis of Bianchi identities is equivalent to the analysis of the cohomology of $\sigma_{-}$, that is

$$
H\left(\sigma_{-}, V\right) \equiv \frac{\operatorname{Ker}\left(\sigma_{-}\right)}{\operatorname{Im}\left(\sigma_{-}\right)} .
$$

The field equation (B.1) is invariant under the gauge transformations

$$
\delta \mathcal{C}=\widehat{D}_{0} \varepsilon
$$

since $\widehat{D}_{0}$ is nilpotent by the hypothesis (iv). The gauge parameter $\varepsilon$ is a $(p-1)$ form. These gauge transformations contain both differential gauge transformations (like linearized diffeomorphisms) and Stueckelberg gauge symmetries (like linearized local Lorentz transformations ${ }^{8}$ ).

The following terminology will be used. By dynamical field, we mean a field that is not expressed as derivatives of something else by field equations (e.g. the frame field in gravity or a frame-like HS 1-form field $e_{m}^{n_{1} \ldots n_{s-1}}$ ). The fields that are expressed by virtue of the field equations as derivatives of the dynamical fields modulo Stueckelberg gauge symmetries are referred to as auxiliary fields (e.g. the Lorentz connection in gravity or its HS analogues $\omega_{m}^{n_{1} \ldots n_{s-1}, m_{1} \ldots m_{t}}$ with $\left.t>0\right)$. A field that is neither auxiliary nor pure gauge by Stueckelberg gauge symmetries is said to be a nontrivial dynamical field (e.g., the metric tensor or the metric-like gauge fields of Fronsdal's approach).

Let $\mathcal{C}(x)$ be a section of the fiber bundle with space-time (coordinate $x$ ) as the base manifold and $V \otimes \Omega^{p}\left(\mathcal{M}^{d}\right)$ as the fibers, that satisfies the equation (B.1). Under the hypotheses (i)-(iv) the following propositions are true [18, 21]:

A. Nontrivial dynamical fields $\mathcal{C}$ are nonvanishing elements of $H^{p}\left(\sigma_{-}\right)$.

B. Differential gauge symmetry parameters $\varepsilon$ are classified by $H^{p-1}\left(\sigma_{-}\right)$.

\footnotetext{
${ }^{8}$ Recall that, at the linearized level, the metric tensor corresponds to the symmetric part $e_{\{\underline{m} m\}}$ of the frame field. The antisymmetric part of the frame field $e_{[\underline{m} m]}$ can be gauged away by fixing locally the Lorentz symmetry, because it contains as many independent components as the Lorentz gauge parameter $\varepsilon^{n m}$.
} 
C. Inequivalent differential field equations on the nontrivial dynamical fields, contained in $\widehat{D}_{0} \mathcal{C}=0$, are in one-to-one correspondence with elements of $H^{p+1}\left(\sigma_{-}\right)$.

Proof of A: The first claim is almost obvious. Indeed, let us decompose the field $\mathcal{C}$ according to the grade $G$ :

$$
\mathcal{C}=\sum_{n=0} \mathcal{C}_{n}, \quad G_{\mathcal{C}_{n}}=n \mathcal{C}_{n}, \quad(n=0,1,2, \ldots) .
$$

The field equation (B.1) thus decomposes as

$$
\left.\widehat{D}_{0} \mathcal{C}\right|_{n-1}=\sigma_{-} \mathcal{C}_{n}+D_{0} \mathcal{C}_{n-1}+\left.\left(\sigma_{+} \sum_{m \leq n-2} \mathcal{C}_{m}\right)\right|_{n-1}=0 .
$$

By a straightforward induction on $n=1,2, \ldots$, one can convince oneself that all fields $\mathcal{C}_{n}$ that contribute to the first term of the right hand side of the equation (B.6) are thereby expressed in terms of derivatives of lower grade $($ i.e. $<n)$ fields, hence they are auxiliary ${ }^{9}$. As a result only fields annihilated by $\sigma_{-}$are not auxiliary. Taking into account the gauge transformation (B.5)

$$
\delta \mathcal{C}_{n}=\left.\widehat{D}_{0} \varepsilon\right|_{n}=\sigma_{-} \varepsilon_{n+1}+D_{0} \varepsilon_{n}+\left.\left(\sigma_{+} \sum_{m \leq n-1} \varepsilon_{m}\right)\right|_{n}
$$

one observes that, due to the first term in this transformation law, all components $\mathcal{C}_{n}$ which are $\sigma_{-}$exact, i.e. which belong to the image of $\sigma_{-}$, are Stueckelberg and they can be gauged away. Therefore, a nontrivial dynamical $p$-form field in $\mathcal{C}$ should belong to the quotient $\operatorname{Ker}\left(\sigma_{-}\right) / \operatorname{Im}\left(\sigma_{-}\right)$

For Einstein-Cartan's gravity, the Stueckelberg gauge symmetry is the local Lorentz symmetry and indeed what distinguishes the frame field from the metric tensor is that the latter belongs to the cohomology $H^{1}\left(\sigma_{-}\right)$while the former contains a $\sigma_{-}$exact part.

Proof of B: The proof follows the same lines as the proof of A. The first step has already been performed in the sense that (B.7) already told us that the parameters such that $\sigma_{-} \varepsilon \neq 0$ are Stueckelberg and can be used to completely gauge away trivial parts of the field $\mathcal{C}$. Thus differential parameters must be $\sigma_{-}$closed. The only subtlety is that one should make use of the fact that

\footnotetext{
${ }^{9}$ Here we use the fact that the operator $\sigma_{-}$acts vertically (that is, it does not differentiate space-time coordinates) thus giving rise to algebraic conditions which express auxiliary fields via derivatives of the other fields.
} 
the gauge transformation $\delta_{\varepsilon} \mathcal{C}=\widehat{D}_{0} \varepsilon$ are reducible. More precisely, gauge parameters obeying the reducibility identity

$$
\varepsilon=\widehat{D}_{0} \zeta
$$

are trivial in the sense that they do not perform any gauge transformation, $\delta_{\varepsilon=\widehat{D}_{0} \zeta} \mathcal{C}=0$. The second step of the proof is a mere decomposition of the reducibility identity (B.8) in order to see that $\sigma_{-}$exact parameters correspond to reducible gauge transformations ${ }^{10}$.

Proof of C: Given a nonnegative integer number $n_{0}$, let us suppose that one has already obtained and analyzed (B.1) in grades ranging from $n=0$ up to $n=n_{0}-1$. Let us analyze (B.1) in grade $G$ equal to $n_{0}$ by looking at the constraints imposed by the Bianchi identities. Applying the operator $\widehat{D}_{0}$ on the covariant derivative $\widehat{D}_{0} \mathcal{C}$ gives identically zero, which is the Bianchi identity $\left(\widehat{D}_{0}\right)^{2} \mathcal{C}=0$. Decomposing the latter Bianchi identity gives, in grade equal to $n_{0}-1$,

$$
\left.\left(\widehat{D}_{0}\right)^{2} \mathcal{C}\right|_{n_{0}-1}=\sigma_{-}\left(\left.\widehat{D}_{0} \mathcal{C}\right|_{n_{0}}\right)+D_{0}\left(\left.\widehat{D}_{0} \mathcal{C}\right|_{n_{0}-1}\right)+\left.\left(\left.\sigma_{+} \sum_{m \leq n_{0}-2} \widehat{D}_{0} \mathcal{C}\right|_{m}\right)\right|_{n_{0}-1}=0
$$

By the induction hypothesis, the equations $\left.\widehat{D}_{0} \mathcal{C}\right|_{m}=0$ with $m \leq n_{0}-1$ have already been imposed and analyzed. Therefore (B.9) leads to

$$
\sigma_{-}\left(\left.\widehat{D_{0}} \mathcal{C}\right|_{n_{0}}\right)=0
$$

In other words, $\left.\widehat{D}_{0} \mathcal{C}\right|_{n_{0}}$ belongs to $\operatorname{Ker}\left(\sigma_{-}\right)$. Thus it can contain a $\sigma_{-}$exact part and a nontrivial cohomology part:

$$
\left.\widehat{D}_{0} \mathcal{C}\right|_{n_{0}}=\sigma_{-}\left(E_{n_{0}+1}\right)+F_{n_{0}}, \quad F_{n_{0}} \in H^{p+1}\left(\sigma_{-}\right) .
$$

The exact part can be compensated by a field redefinition of the component $\mathcal{C}_{n_{0}+1}$ which was not treated before (by the induction hypothesis). More precisely, if one performs

$$
\mathcal{C}_{n_{0}+1} \rightarrow \mathcal{C}_{n_{0}+1}^{\prime}:=\mathcal{C}_{n_{0}+1}-E_{n_{0}+1}
$$

then one is left with $\left.\widehat{D}_{0} \mathcal{C}^{\prime}\right|_{n_{0}}=F_{n_{0}}$. The field equation (B.1) in grade $n_{0}$ is $\left.\widehat{D}_{0} \mathcal{C}^{\prime}\right|_{n_{0}}=0$. This not only expresses the auxiliary $p$-forms $\mathcal{C}_{n_{0}+1}^{\prime}$ (that

\footnotetext{
${ }^{10}$ Note that factoring out the $\sigma_{-}$exact parameters accounts for algebraic reducibility of gauge symmetries. The gauge parameters in $H^{p-1}\left(\sigma_{-}\right)$may still have differential reducibility analogous to differential gauge symmetries for nontrivial dynamical fields.
} 
are not annihilated by $\left.\sigma_{-}\right)$in terms of derivatives of lower grade $p$-forms $\mathcal{C}_{k}$ $\left(k \leq n_{0}\right)$, but also sets $F_{n_{0}}$ to zero. If the cohomology is non-zero, this imposes some $\mathcal{C}_{n_{0}+1}$-independent conditions on the derivatives of the fields $\mathcal{C}_{k}$ with $k \leq n_{0}$, thus leading to differential restrictions on the nontrivial dynamical fields. Therefore, to each representative of $H^{p+1}\left(\sigma_{-}\right)$corresponds a differential field equation.

Note that if $H^{p+1}\left(\sigma_{-}\right)=0$, the equation (B.1) contains only constraints which express auxiliary fields via derivatives of the dynamical fields, imposing no restrictions on the latter. If $D_{0}$ is a first order differential operator and if $\sigma_{+}$ is at most a second order differential operator (which is true in HS applications) then, if $H^{p+1}\left(\sigma_{-}\right)$is nonzero in the grade $k$ sector, the associated differential equations on a grade $\ell$ dynamical field are of order $k+1-\ell$.

\section{References}

[1] M. A. Vasiliev, Phys. Lett. B238 (1990) 305; Phys. Lett. B285 (1992) 225.

[2] M. A. Vasiliev, Phys. Lett. B567 (2003) 139 [hep-th/0304049].

[3] M. A. Vasiliev, Ann. Phys. (N.Y.) 190 (1989) 59.

[4] M. A. Vasiliev, Nucl. Phys. B324 (1989) 503.

[5] A. Sagnotti, E. Sezgin and P. Sundell, "On Higher Spins with a Strong $\operatorname{Sp}(2, R)$ Condition", [hep-th/0501156].

[6] X. Bekaert, S. Cnockaert, C. Iazeolla and M.A.Vasiliev, Nonlinear Higher Spin Theories in Various Dimensions, hep-th/0503128.

[7] E. S. Fradkin and M. A. Vasiliev, Phys. Lett. B189 (1987) 89; Nucl. Phys. B291 (1987) 141.

[8] M. A. Vasiliev, "Higher spin gauge theories: Star-product and AdS space," in M.A. Shifman ed., The many faces of the superworld (World Scientific), hep-th/9910096.

[9] M. A. Vasiliev, "Higher-Spin Theories and $S p(2 M)$ Invariant SpaceTime", hep-th/0301235. 
[10] T. Lada and J. Stasheff, Internat. J. Theoret. Phys. 32 (1993) 1087, [hep-th/9209099].

[11] G. Barnich and M. Grigoriev, "BRST Extension of the Non-Linear Unfolded Formalism", hep-th/0504119.

[12] R.D'Auria and P. Fre, Nucl. Phys. B201 (1982) 101 [Erratum-ibid. B206 (1982) 496.];

P. van Nieuwenhuizen, "Free Graded Differential Superalgebras," in M. Serdaroglu and E. Inönü ed., Group Theoretical Methods in Physics: Proceedings, Lecture Notes in Physics, Vol.180 (Springer-Verlag, 1983) ;

R. D'Auria, P. Fre, P. K. Townsend and P. van Nieuwenhuizen, Annals Phys. 155 (1984) 423.

[13] D. Sullivan, Publ. Math. IHÉS 47 (1977) 269.

[14] M. Alexandrov, M. Kontsevich, A. Schwarz and O. Zaboronsky, Int. J. Mod. Phys. A12 (1997) 1405, hep-th/9502010.

[15] I.Batalin and G.Vilkovisky, Phys. Lett. B102 (1981) 27; Phys. Rev. D29 (1983) 2567.

[16] G. Barnich, M. Grigoriev, A. Semikhatov and I. Tipunin, "Parent field theory and unfolding in BRST first-quantized terms," hep-th/0406192.

[17] M. A. Vasiliev, Class. Quant. Grav. 11 (1994) 649.

[18] O.V. Shaynkman and M.A. Vasiliev, Theor. Math. Phys. 123 (2000) 683 (p. 323 in the Russian issue), hep-th/0003123.

[19] M. A. Vasiliev, Phys. Lett. B257 (1991) 111.

[20] M.A. Vasiliev, " Relativity, Causality, Locality, Quantization and Duality in the $S p(2 M)$ Invariant Generalized Space-Time", hep-th/0111119; Contribution to the Marinov's Memorial Volume, M.Olshanetsky and A.Vainshtein Eds, World Scientific.

[21] M.A. Vasiliev, Phys. Rev. D66 (2002): 066006, hep-th/0106149.

[22] J. Engquist, E. Sezgin and P. Sundell, Nucl. Phys. B664 (2003) 439 [hep-th/0211113].

[23] S. F. Prokushkin and M. A. Vasiliev, Nucl. Phys. B545 (1999) 385 [hep-th/9806236]. 
[24] F.A. Berends, G.J.Burgers and H. van Dam, Nucl. Phys. B271 (1986) 429.

[25] D. Anselmi, Nucl. Phys. B541 (1999) 323 [hep-th/9808004]; Class. Quant. Grav. 17 (2000) 1383 [hep-th/9906167].

[26] S.E. Konstein, M.A. Vasiliev and V.N. Zaikin, JHEP 0012 (2000) 018 [hep-th/0010239].

[27] B. Fedosov, J. Diff. Geometry 40 (1994) 213.

[28] M. Bordemann, N. Neumaier and S. Waldmann, q-alg/9911016.

[29] C. Cutler and R. Wald, Class. Quant. Grav. 4 (1987) 1267;

N. Boulanger, T. Damour, L. Gualtieri, and M. Henneaux, Nucl. Phys. B597 (2001) 127, hep-th/0007220.

[30] E.A. Ivanov and V.I. Ogievetsky, JETP Lett. 23 (1976) 661.

[31] E. Witten, Phys. Lett. B77 (1978) 394.

[32] P. Yasskin, J. Iesenberg and P. Green, Phys. Lett. B78 (1978) 462.

[33] E.A. Ivanov, JETP Lett. 30 (1979) 452.

[34] A. Pashnev, "Nonlinear realizations of the (super)diffemorphism groups, geometrical objects and integral invariants in the superspace", hep-th/9704203.

[35] M.A. Vasiliev, Fortsch. Phys. 35 (1987) 741.

[36] V.E. Lopatin and M.A. Vasiliev, Mod. Phys. Lett. A3 (1988) 257.

[37] M.A. Vasiliev, Nucl. Phys. B616 (2001) 106, [hep-th/0106200].

[38] B. de Wit and D.Z. Freedman, Phys. Rev. D21 (1980) 358.

[39] C. Fronsdal, Phys. Rev. D18 (1978) 3624; D20 (1979) 848.

[40] D. Sorokin, "Introduction to the classical theory of higher spins," hep-th/0405069;

N. Bouatta, G. Compere, A. Sagnotti, "An Introduction to Free HigherSpin Fields," to appear in the proceedings of the First Solvay Workshop on Higher-Spin Gauge Theories (Brussels, May 2004) hep-th/0409068. 
[41] C. Aragone and S. Deser, Phys. Lett. B86 (1979)161.

[42] D. Francia and A. Sagnotti, Phys. Lett. B543 (2002) 303, [hep-th/0207002]; Class. Quant. Grav. 20 (2003) S473, [hep-th/0212185];

X. Bekaert and N. Boulanger, Commun. Math. Phys. 245 (2004) 27 [hep-th/0208058]; Phys. Lett. B561 (2003) 183, [hep-th/0301243]; P. de Medeiros and C. Hull, JHEP 0305 (2003) 019, [hep-th/0303036].

[43] N. Seiberg and E. Witten, JHEP 09 (1999) 032, [hep-th/9908142].

[44] M. A. Vasiliev, JHEP 12 (2004) 046, [hep-th/0404124].

[45] I. Bars, I. Kishimoto and Y. Matsuo, Phys. Rev D67 (2003) 066002, [hep-th/0302151]. 